\title{
Switchable Product Selectivity in Diazoalkane Coupling Catalyzed by a Two-Coordinate Cobalt Complex
}

\section{Supporting Information}

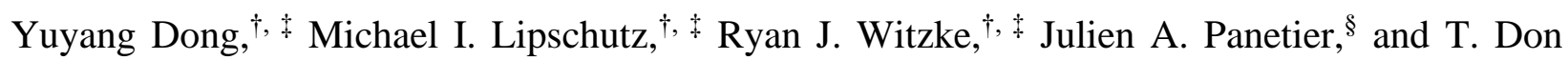
Tilley ${ }^{\dagger,+}$

'Department of Chemistry, University of California, Berkeley, Berkeley, California 94720, United States

*Chemical Sciences Division, Lawrence Berkeley National Laboratory, Berkeley, California 94720, United States

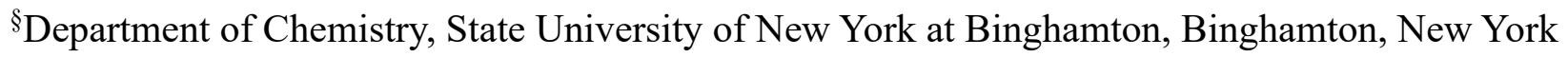
13902, United States

*T. Don Tilley - Email: tdtilley@berkeley.edu

*Julien A. Panetier - Email: panetier@binghamton.edu 


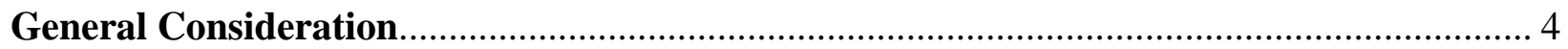

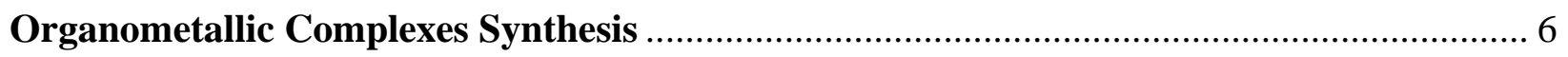

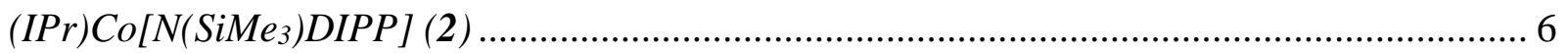

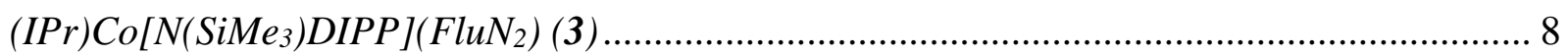

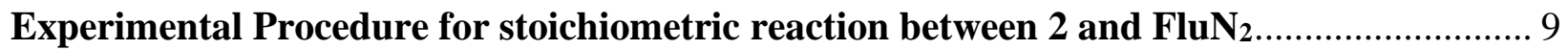

Experimental Procedure for Catalytic Coupling of FluN2 ………………………............... 12

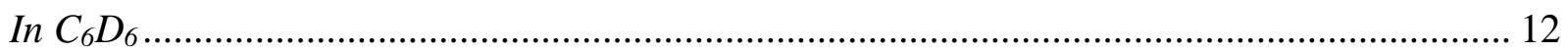

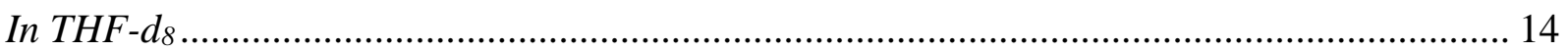

Experimental Procedure for Kinetic Analysis of Homocoupling of FluN2 .......................... 17

Reaction order in substrate concentration ...................................................................... 17

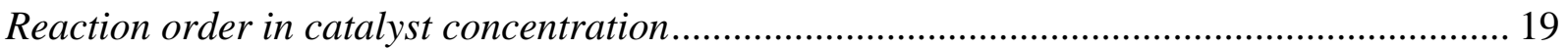

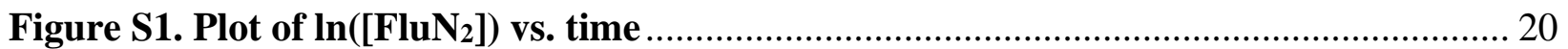

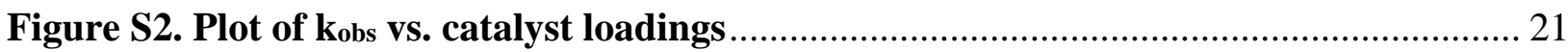

Figure S3. Molecular Orbital Analysis for Reactivity Discrepancy in Benzene with or

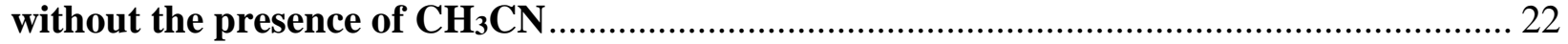

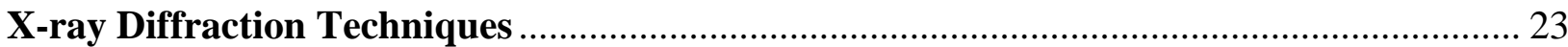

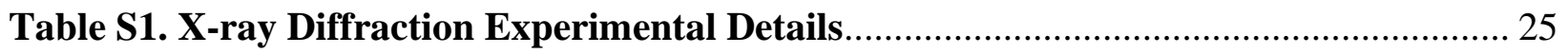

Figure S4. Solid-state structure of (IPr)Co[N(SiMes)DIPP] (2) .......................................... 26

Figure S5. Solid-state structure of (IPr)Co(FluN2)[N(SiMe3)DIPP] (3) ............................ 27

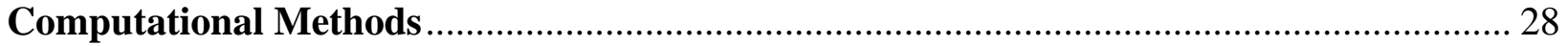

Figure S6. Isosurface (0.004 au) plot of the Mulliken spin population for $3(S=1)$............ 29

Figure S7. Isosurface ( $0.004 \mathrm{au})$ plots of the maximally paired electrons for the $\alpha$ and $\beta$

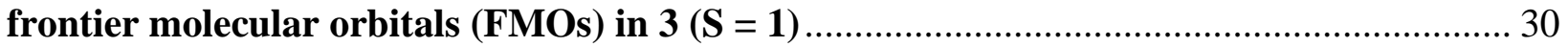

Figure S8. Isosurface ( 0.07 au) plots of the Pipek-Mezey localized d-electrons ( $\alpha$ - and $\beta$ -

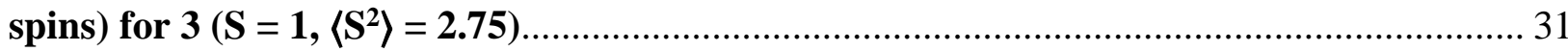

Figure S9. DFT calculated pathway for converting FluN 2 to 9 in the presence of $\mathrm{CH}_{3} \mathrm{CN}$. 32 Figure S10. Transition state for the formation of 8 in the presence of $\mathrm{CH}_{3} \mathrm{CN}$.................. 33

Figure S11. Isosurface ( 0.004 au) plot of the Mulliken spin population for $\operatorname{TS}(3-10)(S=1)$

Figure S12. Isosurface (0.004 au) plot of the Mulliken spin population for $\operatorname{TS}(3-11)(S=1)$ 
Figure S13. Isosurface (0.004 au) plot of the Mulliken spin population for TS(12-13) $(S=1)$ 36

Figure S14. Isosurface (0.004 au) plot of the Mulliken spin population for TS(13-14) $(S=1)$ 36

Table S2. Steps considered to calculate the TOF and $X_{\text {TOF }}$ for the formation of 8 in the presence of 2 and FluN2.....

Table S3. $\mathrm{X}_{\text {TOF }}$ of each intermediate and transition state for the formation of 8 in the presence of 2 and FluN2

Table S4. Steps considered to calculate the TOF and $X_{\text {TOF }}$ for the formation of 9 in the presence of 2 and FluN 2 ......

Table S5. $\mathrm{X}_{\mathrm{TOF}}$ of each intermediate and transition state for the formation of 9 in the presence of 2 and FluN 2

Table S6. Steps considered to calculate the TOF and $\mathrm{X}_{\mathrm{TOF}}$ for the formation of 8 in the presence of $2, \mathrm{FluN}_{2}$ and $\mathrm{CH}_{3} \mathrm{CN}$.

Table S7. X presence of $2, \mathrm{FluN}_{2}$ and $\mathrm{CH}_{3} \mathrm{CN}$.

Table S8. Steps considered to calculate the TOF and $\mathrm{X}_{\text {TOF }}$ for the formation of 9 in the presence of 2, $\mathrm{FluN}_{2}$ and $\mathrm{CH}_{3} \mathrm{CN}$.

Table S9. $\mathrm{X}_{\mathrm{TOF}}$ of each intermediate and transition state for the formation of 9 in the presence of 2, $\mathrm{FluN}_{2}$ and $\mathrm{CH}_{3} \mathrm{CN}$.

Table S10. Steps considered to calculate the TOF and $X_{\text {TOF }}$ for the formation of 8 in the presence of 2 and FluN 2 in THF

Table S11. XтоF of each intermediate and transition state for the formation of 8 in the presence of 2 and FluN 2 in THF

Table S12. Steps considered to calculate the TOF and $X_{\text {TOF }}$ for the formation of 9 in the presence of 2 and FluN 2 in THF

Table S13. $\mathrm{X}_{\text {TOF }}$ of each intermediate and transition state for the formation of 9 in the presence of 2 and FluN 2 in THF

Table S14. Calculated relative energies for 2 at the $\omega$ B97X-D/BS2(SMD)// $\omega$ B97X-D/BS1 level of theory for different spin states.

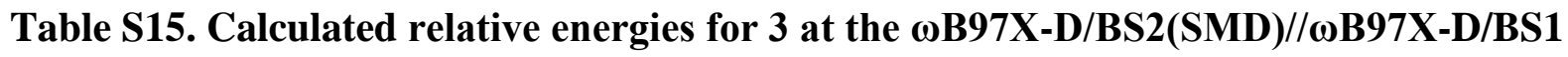

level of theory for different spin states. 45

References 46 


\section{General Consideration}

Unless otherwise stated, all reactions and manipulations were carried out in a MBraun Lab Master DP glovebox or using standard Schlenk techniques under a nitrogen atmosphere. Pentane, toluene, and hexamethyldisiloxane were purchased from Sigma Aldrich. Diethyl ether was purchased from Honeywell and tetrahydrofuran was purchased from Macron Chemicals. Pentane, toluene, tetrahydrofuran and diethyl ether were dried and degassed using a JC Meyers Phoenix SDS solvent purification system. Hexamethyldisiloxane was dried by stirring over potassium metal for 2 days and degassed by four freeze-pump-thaw cycles. Benzene- $d_{6}$ was purchased from Cambridge Isotope Laboratories, dried over $\mathrm{Na} / \mathrm{K}$ alloy and then degassed by four freeze-pump-thaw cycles. All NMR spectra were collected at ambient temperature (ca. $22^{\circ} \mathrm{C}$ ) on Bruker AVB-400, AV-500, AV-600 or AVQ-400 NMR spectrometers, each equipped with a $5 \mathrm{~mm} \mathrm{BB}$ probe, and referenced to the residual proteo solvent signals. Solution magnetic susceptibilities were determined by ${ }^{1} \mathrm{H}$ NMR spectroscopy using Evans's method. ${ }^{1}$ Elemental analyses were performed by the UC Berkeley College of Chemistry Microanalytical facility. The abbreviation "DIPP" refers to a 2,6diisopropylphenyl moiety. The abbreviation "IPr" refers to the N-heterocyclic carbene N,N'-1,3bis(2,6-diisopropylphenyl)imidazol-2-ylidene. The abbreviation "HMDSO" refers to hexamethyldisiloxane. $\mathrm{NEt}_{3} \cdot \mathrm{HCl}$ was purchased from Sigma Aldrich and sublimed before use. $\mathrm{CoCl}_{2}$ was purchased from Strem Chemical and used as received. 9-Diazofluorene, IPr, $\mathrm{LiN}\left(\mathrm{SiMe}_{3}\right) \mathrm{DIPP}$, potassium graphite, and $\mathrm{Co}\left[\mathrm{N}\left(\mathrm{SiMe}_{3}\right) \mathrm{DIPP}\right]_{2}$ were prepared according to literature procedures. ${ }^{2}$ Similar reactions were attempted using several $a$-diazo- $\beta$-ketoester species with the $\mathrm{Co}(\mathrm{I})$ pre-catalyst (2), but only facile catalyst decomposition was observed with no productive transformation of the diazoalkanes. We hypothesized that the conjugation with carbonyl functionalities significantly altered the redox potential of the diazo unit, leading to

\section{Page S4}


catalyst decomposition into several untraceable Co-containing species by ${ }^{1} \mathrm{H}$ NMR spectroscopy. We focused our study on $\mathrm{FluN}_{2}$ due to its enhanced stability among other diazoalkanes. Alkyl substituted diazoalkanes were avoided due to safety precautions.

\section{Page S5}




\section{Organometallic Complexes Synthesis}

$(\mathrm{IPr}) \mathrm{Co}\left[N\left(\mathrm{SiMe}_{3}\right) \mathrm{DIPP}\right](\mathbf{2})$

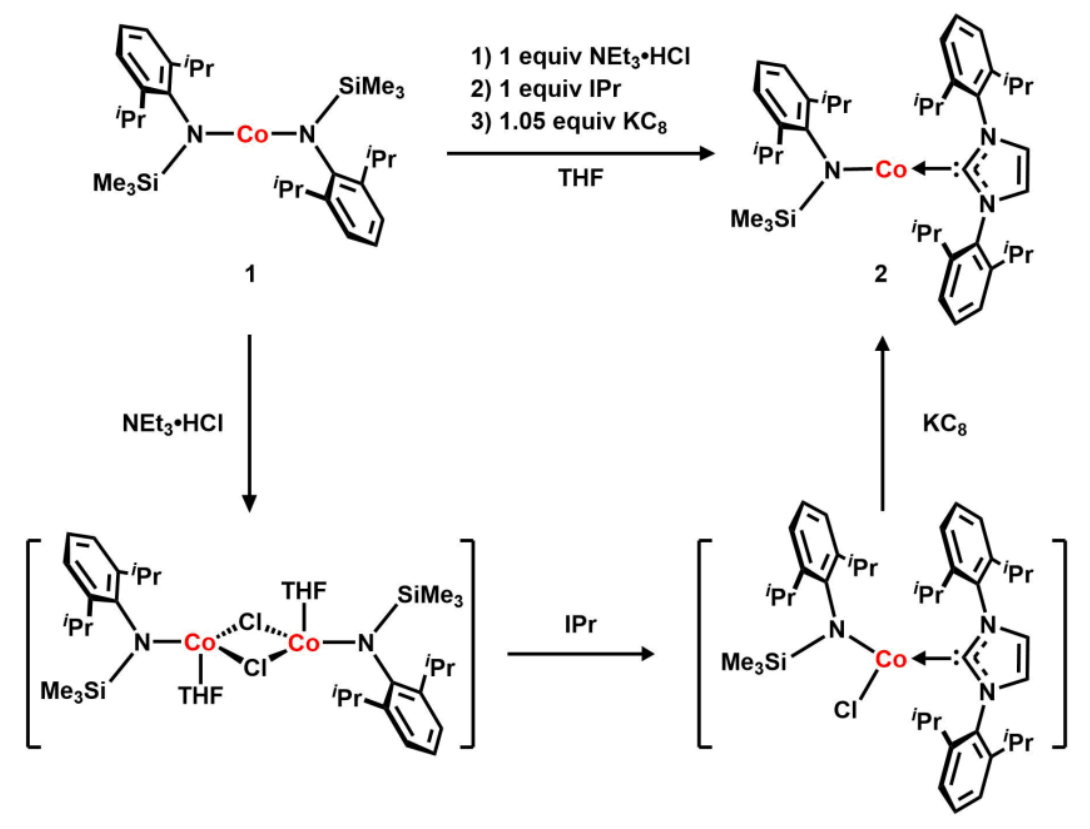

To a $20 \mathrm{~mL}$ scintillation vial was added $\mathrm{Co}\left[\mathrm{N}\left(\mathrm{SiMe}_{3}\right) \mathrm{DIPP}\right]_{2}(\mathbf{1}, 0.400 \mathrm{~g}, 0.720 \mathrm{mmol})$ and $6 \mathrm{~mL}$

of THF to form a green solution. To this stirred solution was added $\mathrm{NEt}_{3} \bullet \mathrm{HCl}(0.099 \mathrm{~g}, 0.719$ mmol) as a solid. Over the course of 5 minutes, the $\mathrm{NEt}_{3} \cdot \mathrm{HCl}$ dissolved, and the solution changed color from green to turquoise. To this stirred, the turquoise solution was then added $\operatorname{IPr}(0.280 \mathrm{~g}$, $0.721 \mathrm{mmol}$ ) in $3 \mathrm{~mL}$ of THF, dropwise. Upon completion of the addition, the solution had changed color from turquoise to yellow. The solution was stirred for 5 additional minutes, then potassium graphite ( $0.107 \mathrm{~g}, 0.792 \mathrm{mmol}, 1.1$ equiv) was added as a solid, causing an immediate color change from yellow to orange. The resulting mixture was stirred for 20 minutes before the stirring was stopped, and the mixture was allowed to settle. The mixture was then filtered through a pipette fitted with a glass fiber filter into a $20 \mathrm{~mL}$ scintillation vial. The filtrate was then placed under vacuum, and the volatile components were removed. The resulting orange residue was dissolved 
in $5 \mathrm{~mL}$ of toluene and layered with $15 \mathrm{~mL}$ of HMDSO, and the mixture was placed in the freezer at $-30{ }^{\circ} \mathrm{C}$ for $18 \mathrm{~h}$ to yield $0.25 \mathrm{~g}(49 \%)$ of (IPr) $\mathrm{Co}\left[\mathrm{N}\left(\mathrm{SiMe}_{3}\right) \mathrm{DIPP}\right]$ (2) as long, narrow, orange plates that were isolated by decantation and dried under vacuum until fully desolvated, requiring approximately 2 hours to reach constant mass. The supernatant was dried under vacuum and the residue was recrystallized and isolated in an identical fashion using $2 \mathrm{~mL}$ of toluene and $8 \mathrm{~mL}$ of HMDSO, yielding an additional $0.12 \mathrm{~g}$ of $\mathbf{2}$, bringing the total yield to $0.36 \mathrm{~g}(73 \%) .{ }^{1} \mathrm{H}$ NMR spectra were taken with a window between 200 and $-190 \mathrm{ppm}$. The expected number of unique shifts is 12 , but only 11 were located. It is possible the remaining shift is beyond this window or sufficiently broad as not to be detectable. ${ }^{1} \mathrm{H}$ NMR $\left(500 \mathrm{MHz}, \mathrm{C}_{6} \mathrm{D}_{6}, 20^{\circ} \mathrm{C}\right) \delta 178.70,64.08,44.39$, $41.70,22.58,15.69,3.26,-35.15,-44.48,-136.18,-139.11 . \mu_{\text {eff }}=4.8 \mu_{\mathrm{B}}\left(\mathrm{C}_{6} \mathrm{D}_{6}, 20.5^{\circ} \mathrm{C}\right.$, Evans's method). Anal. Calcd. for $\mathrm{C}_{42} \mathrm{H}_{62} \mathrm{CoN}_{3} \mathrm{Si}$ : C, 72.48\%; H, 8.98\%; N, 6.04\%. Found: C, 72.18\%; H, $8.62 \% ; \mathrm{N}, 6.21 \%$. Crystals suitable for single-crystal X-ray diffraction studies were obtained from the workup described above. 


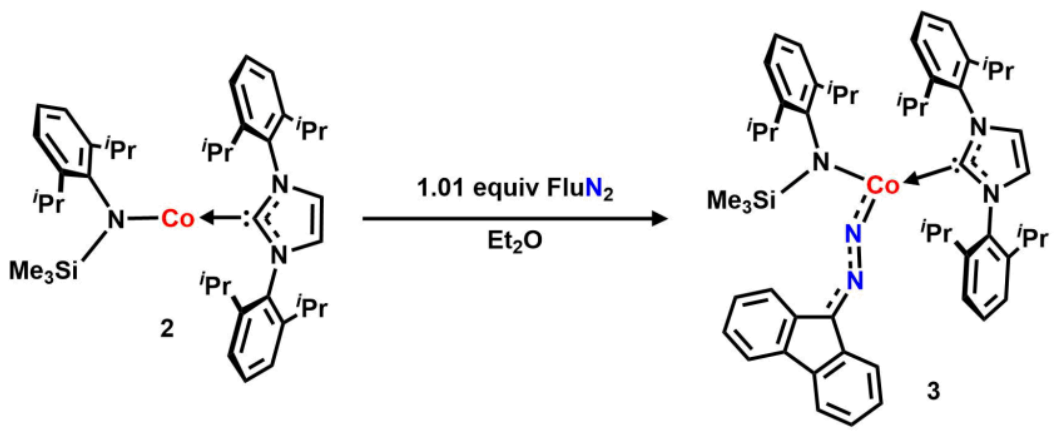

To a $20 \mathrm{~mL}$ scintillation vial was added $2(0.050 \mathrm{~g}, 0.072 \mathrm{mmol})$ and $2 \mathrm{~mL}$ of $\mathrm{Et}_{2} \mathrm{O}$, forming an orange solution. To this stirred solution was added 9-diazofluorene $(0.014 \mathrm{~g}, 0.073 \mathrm{mmol})$ dissolved in $2 \mathrm{~mL}$ of $\mathrm{Et}_{2} \mathrm{O}$, dropwise, by pipette, whereupon the solution changed color from orange to very dark, opaque orange/yellow. After the addition was completed, the reaction mixture was allowed to stir for an additional minute, then vacuum was applied, and the volatile components were removed. The resulting residue was redissolved in $3 \mathrm{~mL}$ of toluene and layered with $8 \mathrm{~mL}$ of pentane. The mixture was placed in the freezer at $-30{ }^{\circ} \mathrm{C}$ for $20 \mathrm{~h}$ to yield $0.052 \mathrm{~g}(81 \%)$ of $(\operatorname{IPr}) \mathrm{Co}\left[\mathrm{N}\left(\mathrm{SiMe}_{3}\right) \mathrm{DIPP}\right]\left(\mathrm{FluN}_{2}\right)(3)$ as dark orange plates. ${ }^{1} \mathrm{H} \mathrm{NMR}\left(400 \mathrm{MHz}, \mathrm{C}_{6} \mathrm{D}_{6}, 20{ }^{\circ} \mathrm{C}\right) \delta$ $40.73(2 \mathrm{H}), 33.25(2 \mathrm{H}), 24.42(2 \mathrm{H}), 20.68(4 \mathrm{H}), 15.53(2 \mathrm{H}), 11.35(2 \mathrm{H}), 10.59(6 \mathrm{H}), 9.20(12 \mathrm{H})$, $4.68(9 \mathrm{H}),-8.96(4 \mathrm{H}),-9.64(2 \mathrm{H}),-12.58(1 \mathrm{H}$, overlapping $),-13.02(12 \mathrm{H}$, overlapping), -33.89 $(6 \mathrm{H}),-35.55(2 \mathrm{H}),-38.01(2 \mathrm{H}) . \mu_{\mathrm{eff}}=2.5 \mu_{\mathrm{B}}\left(\mathrm{C}_{6} \mathrm{D}_{6}, 20^{\circ} \mathrm{C}\right.$, Evans's method $)$. Anal. Calcd. for $\mathrm{C}_{55} \mathrm{H}_{70} \mathrm{CoN}_{5} \mathrm{Si}: \mathrm{C}, 74.37 \% ; \mathrm{H}, 7.94 \% ; \mathrm{N}, 7.88 \%$. Found: C, 74.67\%; H, 8.23\%; N, 7.49\%. Crystals suitable for single-crystal X-ray diffraction studies were obtained from the workup described above.

\section{Page S8}




\section{Experimental Procedure for stoichiometric reaction between 2 and FluN 2}

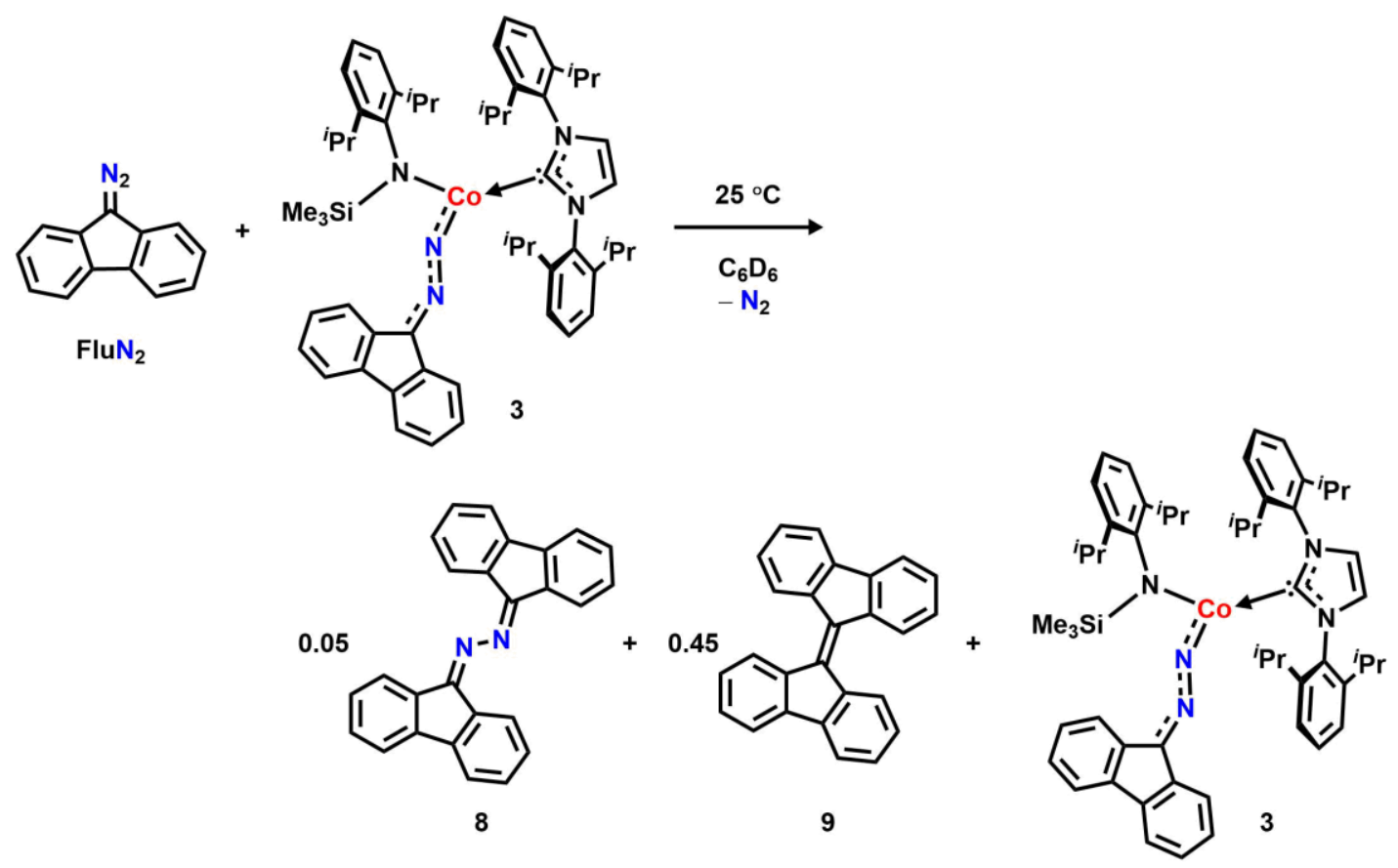

To a $20 \mathrm{~mL}$ scintillation vial was added $\mathbf{3}(20.0 \mathrm{mg}, 0.022 \mathrm{mmol})$ and $0.5 \mathrm{ml}$ of $\mathrm{C}_{6} \mathrm{D}_{6}$, forming an orange solution. To this solution was added 9-diazofluorene $(4.3 \mathrm{mg}, 0.022 \mathrm{mmol})$ dissolved in $0.5 \mathrm{~mL}$ of $\mathrm{C}_{6} \mathrm{D}_{6}$, dropwise, by pipette, whereupon the solution changed color from orange to dark yellow. After the addition was completed, the reaction mixture was transferred to a J-young tube, and effervescence was observed over the course of $20 \mathrm{~min}$. The reaction progress was monitored by ${ }^{1} \mathrm{H}$ NMR spectroscopy, and full conversion of $\mathrm{FluN}_{2}$ was observed after the bubbling has ceased, resulting in the formation of 1,2-di(9H-fluoren-9-ylidene)hydrazine (8) and 9,9'-bifluorenylidene (9) in 10\% and 90\% yield based on final NMR spectrum, respectively. The paramagnetic region of the final ${ }^{1} \mathrm{H}$ NMR spectrum taken for the reaction mixture further showed no consumption of diazoalkane adduct 3 . 


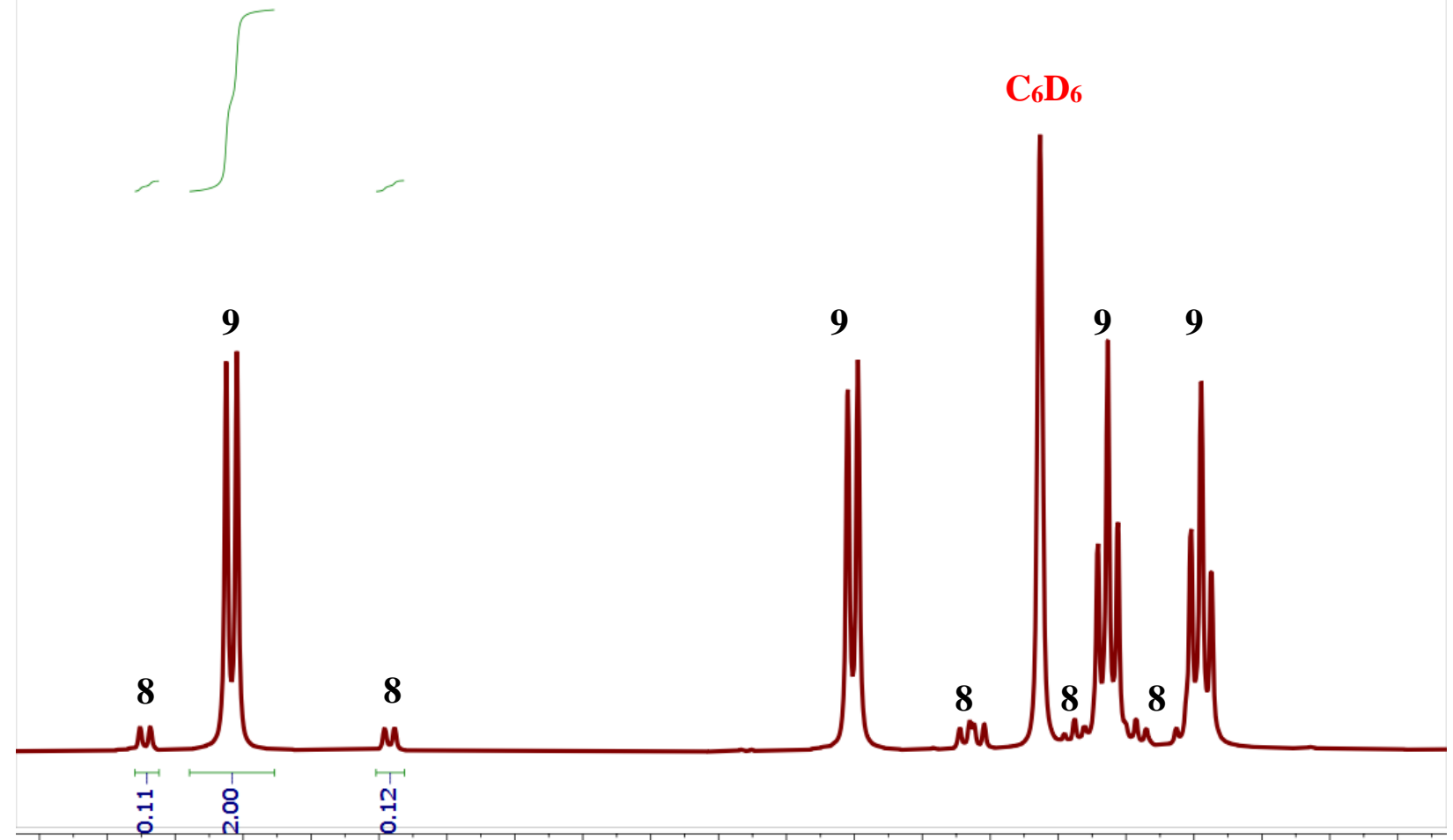

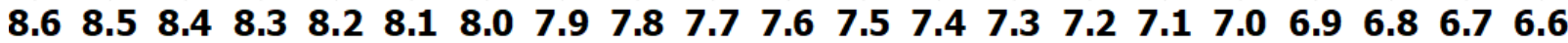
Chemical Shift [ppm]

Diamagnetic region of final ${ }^{1} \mathrm{H}$ NMR spectrum of the reaction mixture

The spectrum shows the full conversion of FluN 2 into hydrazine 8 and olefin 9 in $11 \%$ and 89\% yield, respectively. 


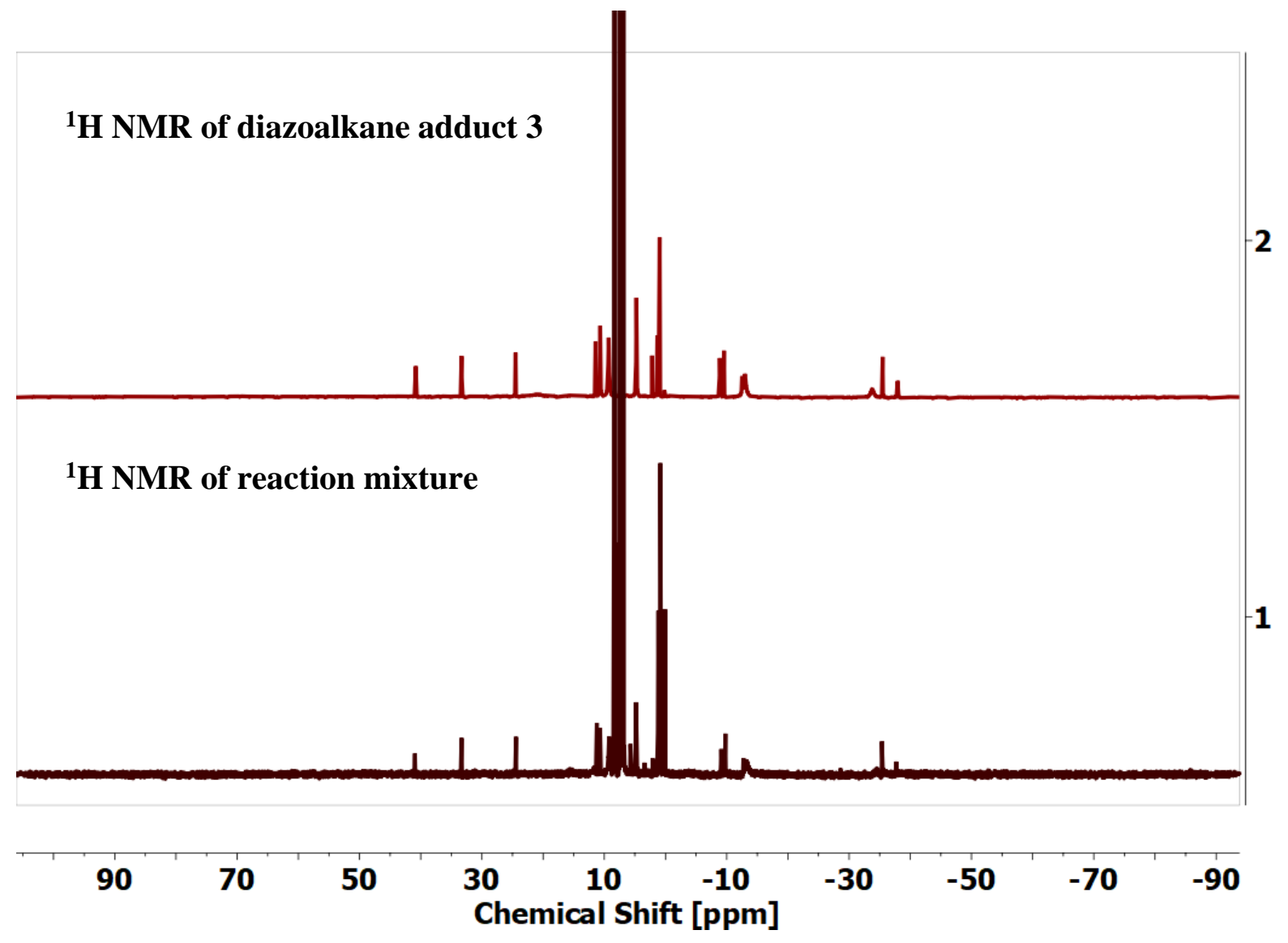

Paramagnetic region of final ${ }^{1} \mathrm{H}$ NMR spectrum for a crystal of 3 and FluN $\mathrm{N}_{2}$ coupling reaction mixture

The spectrum shows the only paramagnetic species as the diazoalkane adduct $\mathbf{3}$. 


\section{Experimental Procedure for Catalytic Coupling of FluN2}

In $C_{6} D_{6}$

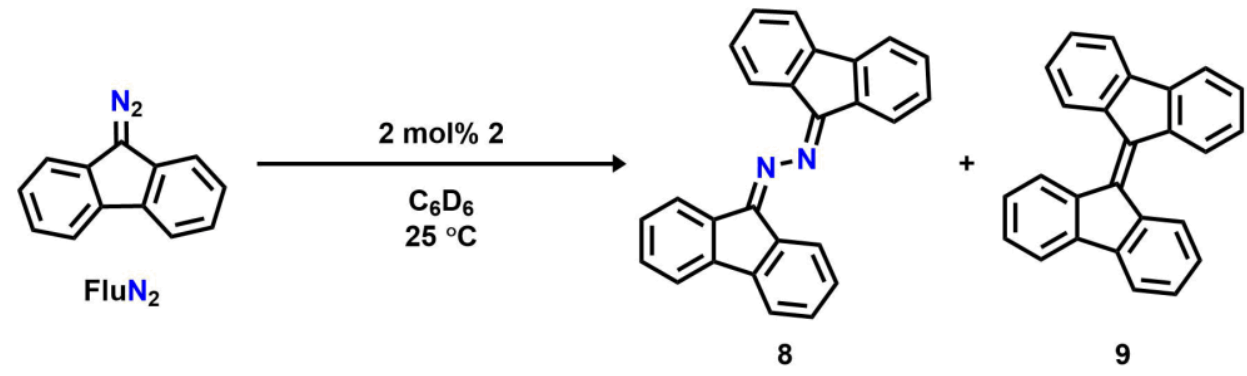

Catalyst 2 stock solution A preparation: To a $20 \mathrm{~mL}$ scintillation vial was added 2 (14.5 mg, 0.021 mmol) followed by $1.0 \mathrm{ml}$ of $\mathrm{C}_{6} \mathrm{D}_{6}$.

To a $20 \mathrm{~mL}$ scintillation vial was added FluN $2(20.0 \mathrm{mg}, 0.104 \mathrm{mmol})$ and $0.5 \mathrm{ml}$ of $\mathrm{C}_{6} \mathrm{D}_{6}$, forming an orange solution. To this solution was added $0.1 \mathrm{ml}$ of catalyst stock solution A (0.002 mmol) dropwise by syringe, whereupon the solution changed color from orange to dark yellow. An additional $0.4 \mathrm{ml}$ of $\mathrm{C}_{6} \mathrm{D}_{6}$ was added to bring the total solution volume to $1.0 \mathrm{ml}$. After the addition was completed, the reaction mixture was transferred to a J-young tube, and effervescence was observed for $3 \mathrm{~h}$. The reaction progress was monitored by ${ }^{1} \mathrm{H}$ NMR spectroscopy, and full conversion of FluN 2 was observed after the bubbling has ceased, resulting in the formation of 1,2di(9H-fluoren-9-ylidene)hydrazine (8) and 9,9'-bifluorenylidene (9) in 11\% and 89\% yield based on final NMR spectrum, respectively. The paramagnetic region of the ${ }^{1} \mathrm{H}$ NMR spectrum taken for the mixture during and after the reaction showed the only observable paramagnetic species as the diazoalkane adduct 3. The ${ }^{1} \mathrm{H}$ NMR spectrum taken resembles the ones from the stoichiometric reaction between 3 and FluN2. 


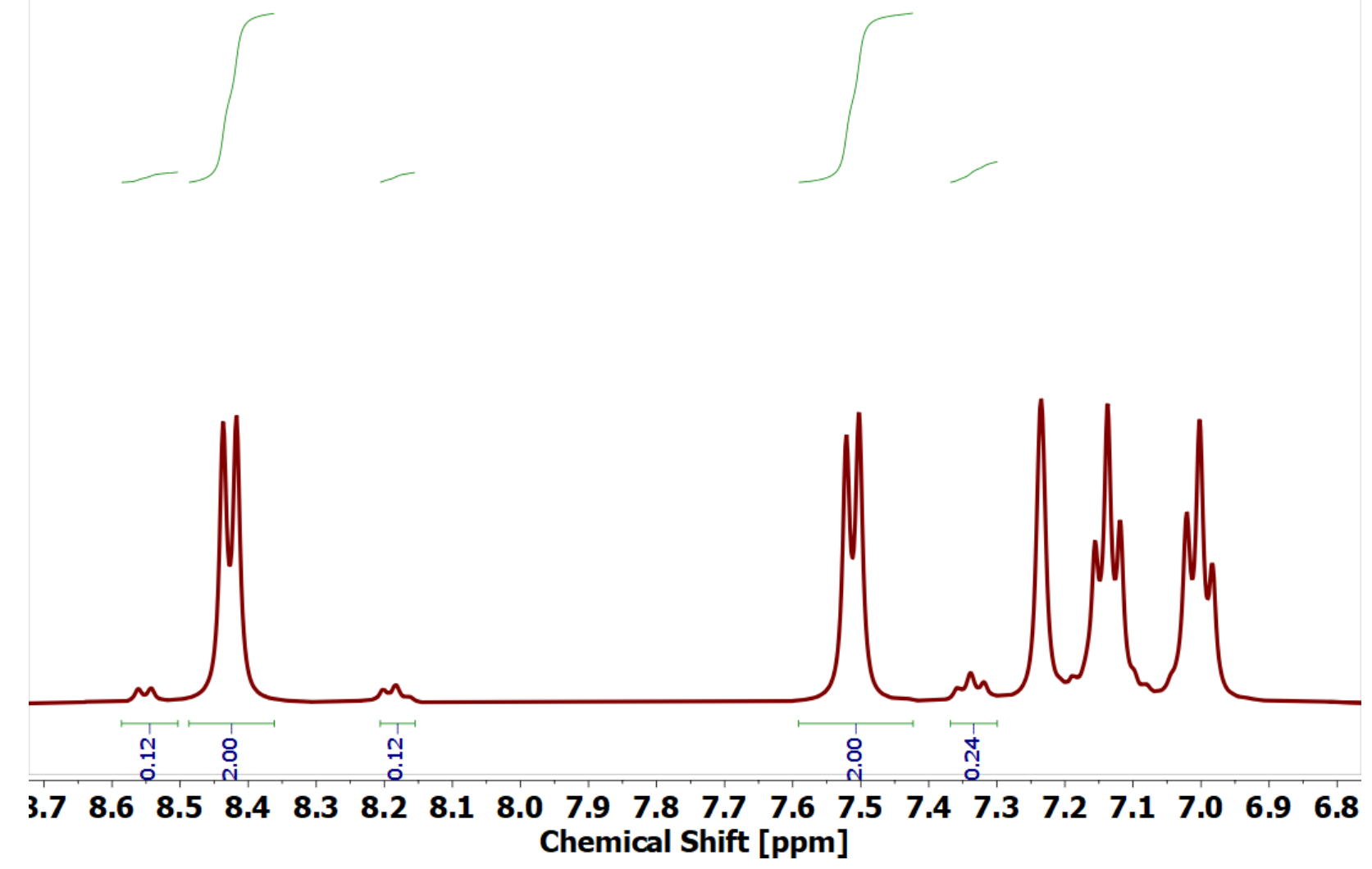

Diamagnetic region of final ${ }^{1} \mathrm{H}$ NMR spectrum of the catalytic reaction mixture 
In $T H F-d_{8}$

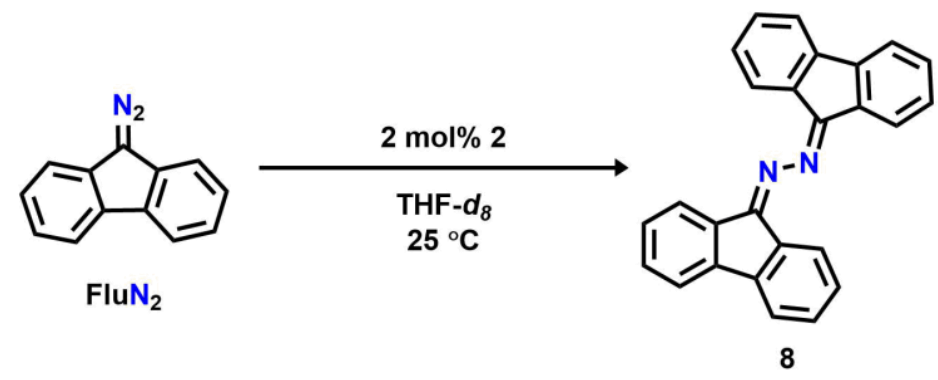

Catalyst 2 stock solution B preparation: To a $20 \mathrm{~mL}$ scintillation vial was added 2 (14.5 $\mathrm{mg}, 0.021$ mmol) followed by $1.0 \mathrm{ml}$ of THF- $d_{8}$.

To a $20 \mathrm{~mL}$ scintillation vial was added FluN $2(20.0 \mathrm{mg}, 0.104 \mathrm{mmol})$ and $0.5 \mathrm{ml}$ of THF-d $\mathrm{d}_{8}$, forming an orange solution. To this solution was added $0.1 \mathrm{ml}$ of catalyst stock solution A (0.002 mmol) dropwise by syringe, whereupon the solution changed color from orange to dark yellow. An additional $0.4 \mathrm{ml}$ of THF- $\mathrm{d}_{8}$ was added to bring the total solution volume to $1.0 \mathrm{ml}$. After the addition was completed, the reaction mixture was transferred to a J-young tube, and effervescence was observed for $3 \mathrm{~h}$. The reaction progress was monitored by ${ }^{1} \mathrm{H}$ NMR spectroscopy, and full conversion of FluN $N_{2}$ was observed after the bubbling has ceased, resulting in the sole formation of 1,2-di(9H-fluoren-9-ylidene)hydrazine (8). The product identity is further confirmed by comparing the ${ }^{1} \mathrm{H}$ NMR features of the product mixture in $\mathrm{CDCl}_{3}$ to literature values. 


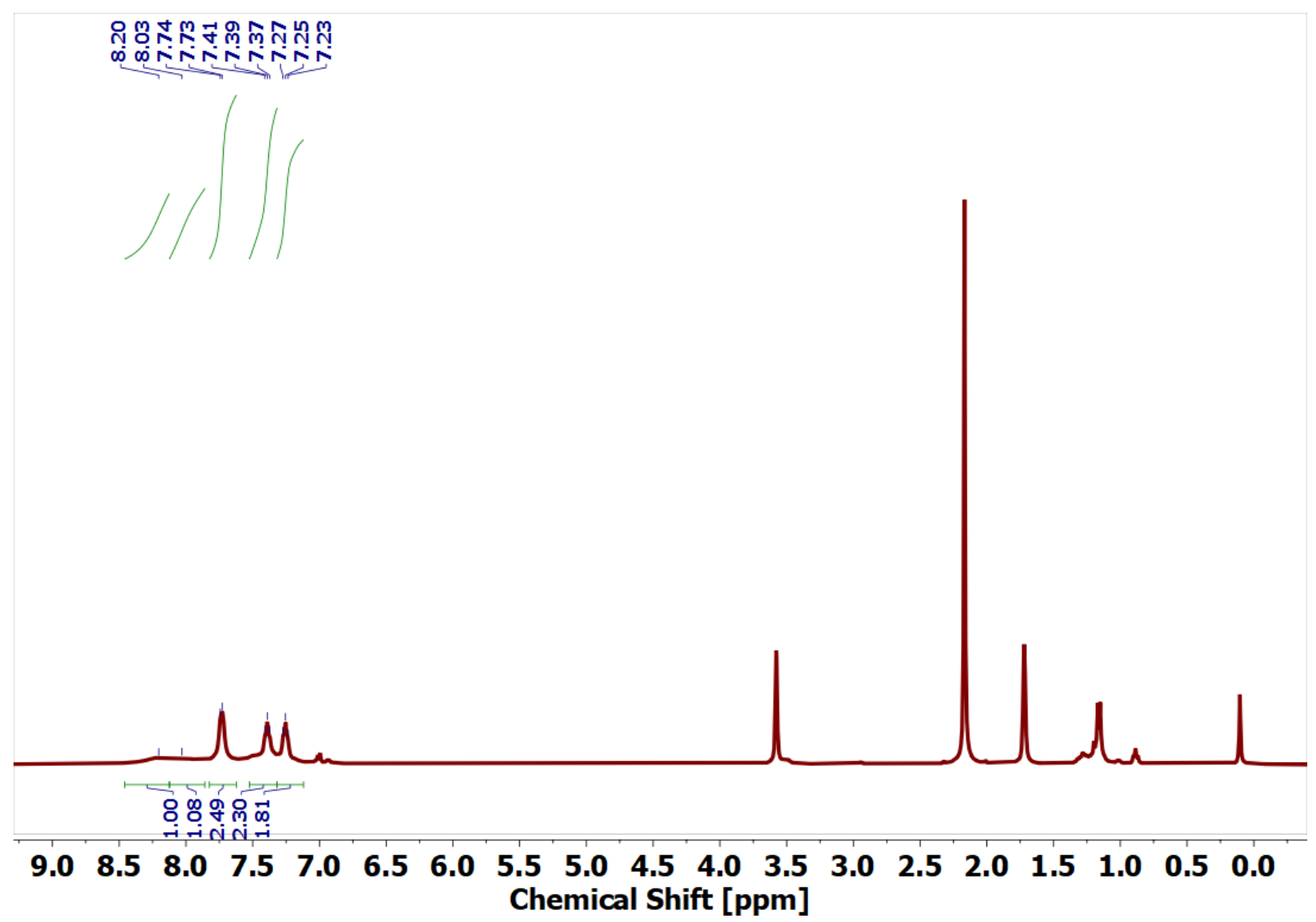

${ }^{1} \mathrm{H}$ NMR spectrum of the reaction mixture in THF- $d_{8}$

The spectrum shows only one species in the aromatic region, but the paramagnetic broadening prevents accurate integration and product assignment. 


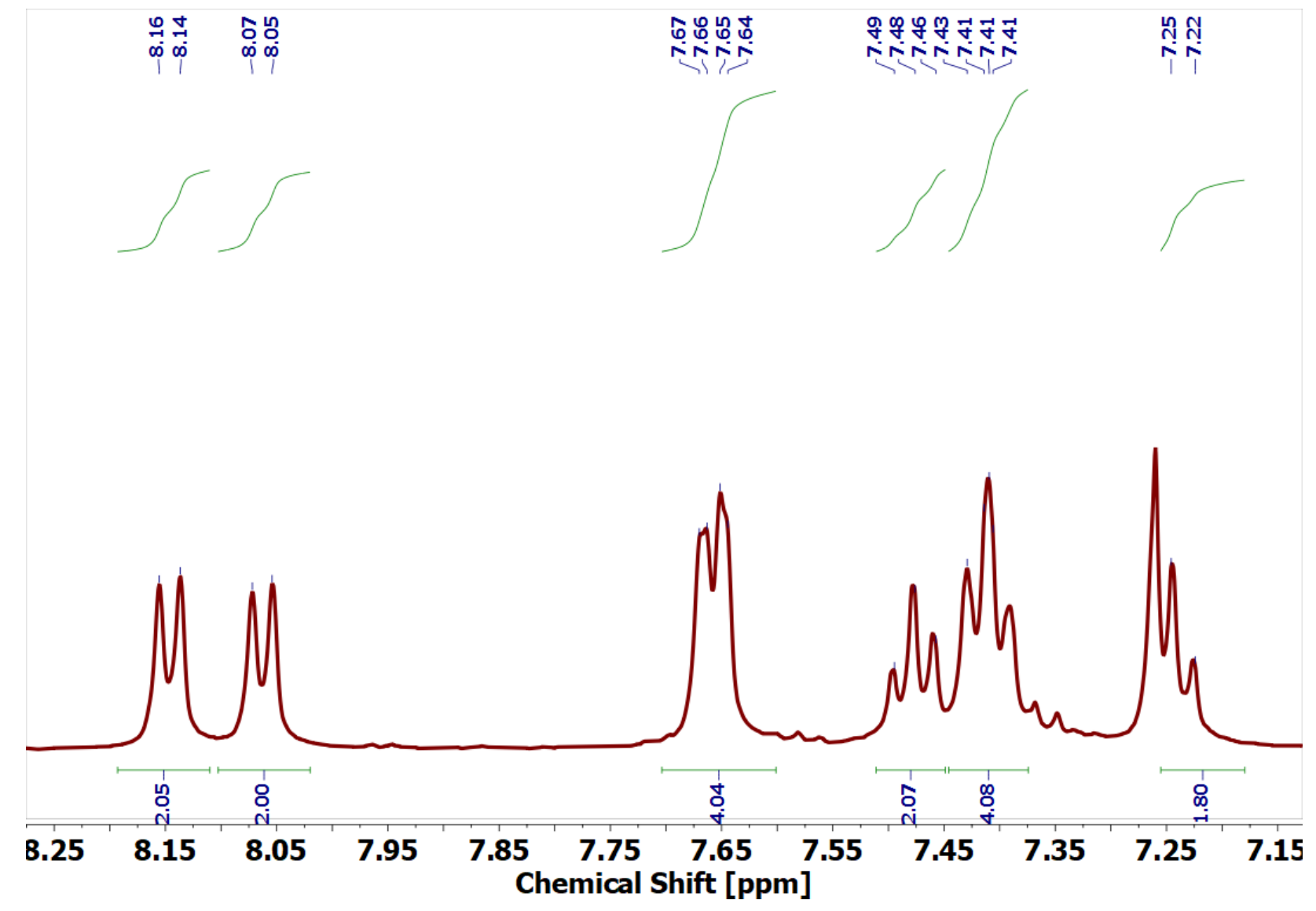

${ }^{1} \mathrm{H}$ NMR spectrum of reaction mixture redissolved in $\mathrm{CDCl}_{3}$

The spectrum shows the one major species in the aromatic region possesses $C_{2 h}$ symmetry (8) rather than $D_{2 h}(9)$. The NMR shifts also match those reported for hydrazine 8. 


\section{Experimental Procedure for Kinetic Analysis of Homocoupling of FluN2}

Reaction order in substrate concentration

Catalyst 2 stock solution C preparation: To a $20 \mathrm{~mL}$ scintillation vial was added $2(0.12 \mathrm{~g}, 0.172$ mmol) followed by $6.0 \mathrm{ml}$ of $\mathrm{C}_{6} \mathrm{D}_{6}$.

Substrate FluN 2 stock solution D preparation: To a $20 \mathrm{~mL}$ scintillation vial was added FluN $2(0.50$ $\mathrm{g}, 2.60 \mathrm{mmol}$ ) and hexamethylbenzene (internal standard, $10.0 \mathrm{mg}$ ) followed by $12.5 \mathrm{ml}$ of $\mathrm{C}_{6} \mathrm{D}_{6}$.

In a $\mathrm{N}_{2}$-filled dry-box, a J-young $\mathrm{NMR}$ tube was added $0.5 \mathrm{ml}$ of stock solution $\mathrm{D}$, and the whole tube was left inside a cold well chilled with liquid nitrogen until the solution is completely frozen. An additional $0.35 \mathrm{ml}$ of $\mathrm{C}_{6} \mathrm{D}_{6}$ was added to the tube and frozen using the same procedure, followed by $0.15 \mathrm{ml}$ of catalyst 2 stock solution $\mathrm{C}$ to bring the total solution volume to $1.0 \mathrm{ml}$. The whole mixture was left in the cold well for 10 min before sealed and taken out of the dry-box. The J-young tube was kept immersed in a dry ice/isopropanol mixture to keep the frozen solution from thawing.

The tube was quickly thawed and shaken before inserted into the NMR instruments for kinetic data acquisition. 
89 FluN $_{2}$

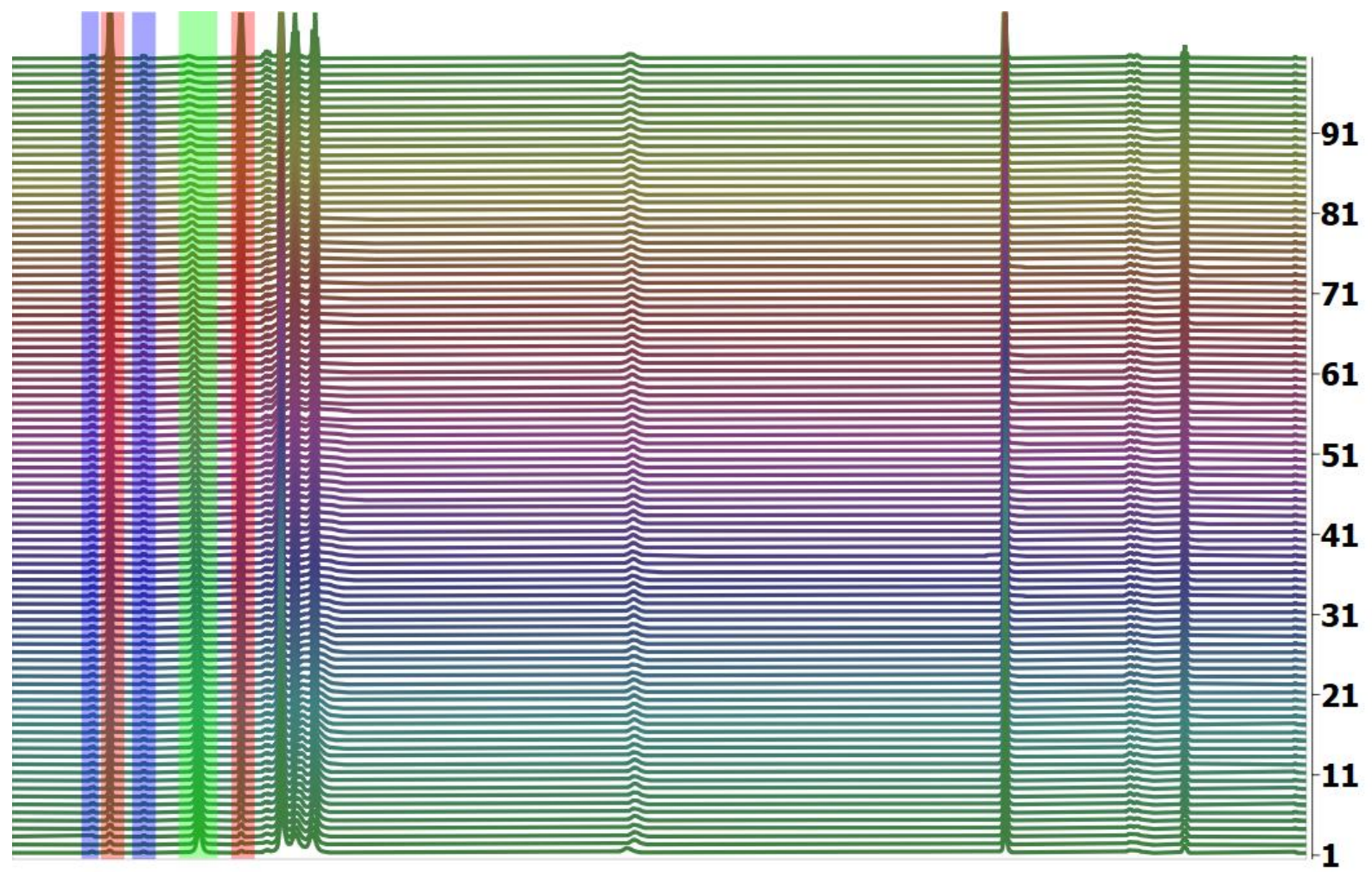

\section{$\begin{array}{lllllllllllllllllllllll}8.5 & 8.0 & 7.5 & 7.0 & 6.5 & 6.0 & 5.5 & 5.0 & 4.5 & 4.0 & 3.5 & 3.0 & 2.5 & 2.0 & 1.5 & 1.0 & 0.5 & 0.0\end{array}$ Chemical Shift [ppm]}

\section{${ }^{1} \mathrm{H}$ NMR stack for FluN $_{2}$ coupling reaction using catalyst 2}

The formation of products 8 and 9 are highlighted using blue and red, respectively. The consumption of FluN 2 is labelled in green. Peak at 2.13 ppm corresponds to the internal standard hexamethylbenzene. 
Reaction order in catalyst concentration

In a $\mathrm{N}_{2}$-filled dry-box, a J-young NMR tube was added $0.5 \mathrm{ml}$ of stock solution $\mathrm{D}$, and the whole tube was left inside a cold well chilled with liquid nitrogen until the solution is completely frozen. An additional amount of $\mathrm{C}_{6} \mathrm{D}_{6}$ was added to the tube and frozen (if necessary) using the same procedure, followed by the desired amount of catalyst $\mathbf{2}$ stock solution $\mathrm{C}$ to bring the total solution volume to $1.0 \mathrm{ml}$. The whole mixture was left in the cold well for $10 \mathrm{~min}$ before sealed and taken out of the dry-box. The J-young tube was kept immersed in a dry ice/isopropanol mixture to keep the frozen solution from thawing.

The tube was quickly thawed and shaken before inserted into the NMR instruments for kinetic data acquisition. Each kinetic run with a specific catalyst concentration is repeated two more times for error bar measurement. 
Figure S1. Plot of $\ln \left(\left[\mathrm{FluN}_{2}\right]\right)$ vs. time

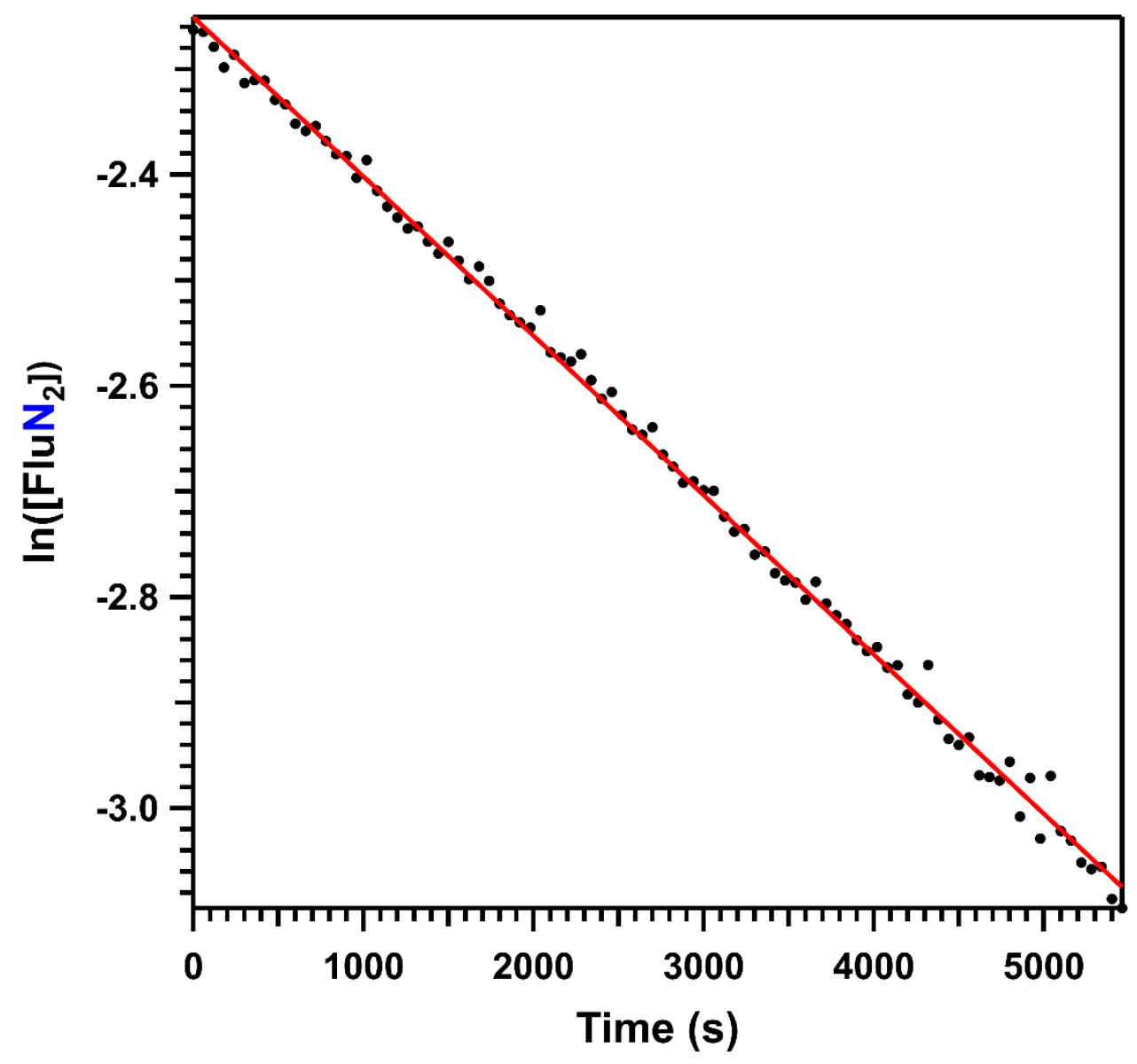

Linear relationship between $\ln \left(\left[\mathrm{FluN}_{2}\right]\right)$ over time suggests a first-order reaction rate dependence on substrate concentration. 
Figure S2. Plot of kobs vs. catalyst loadings

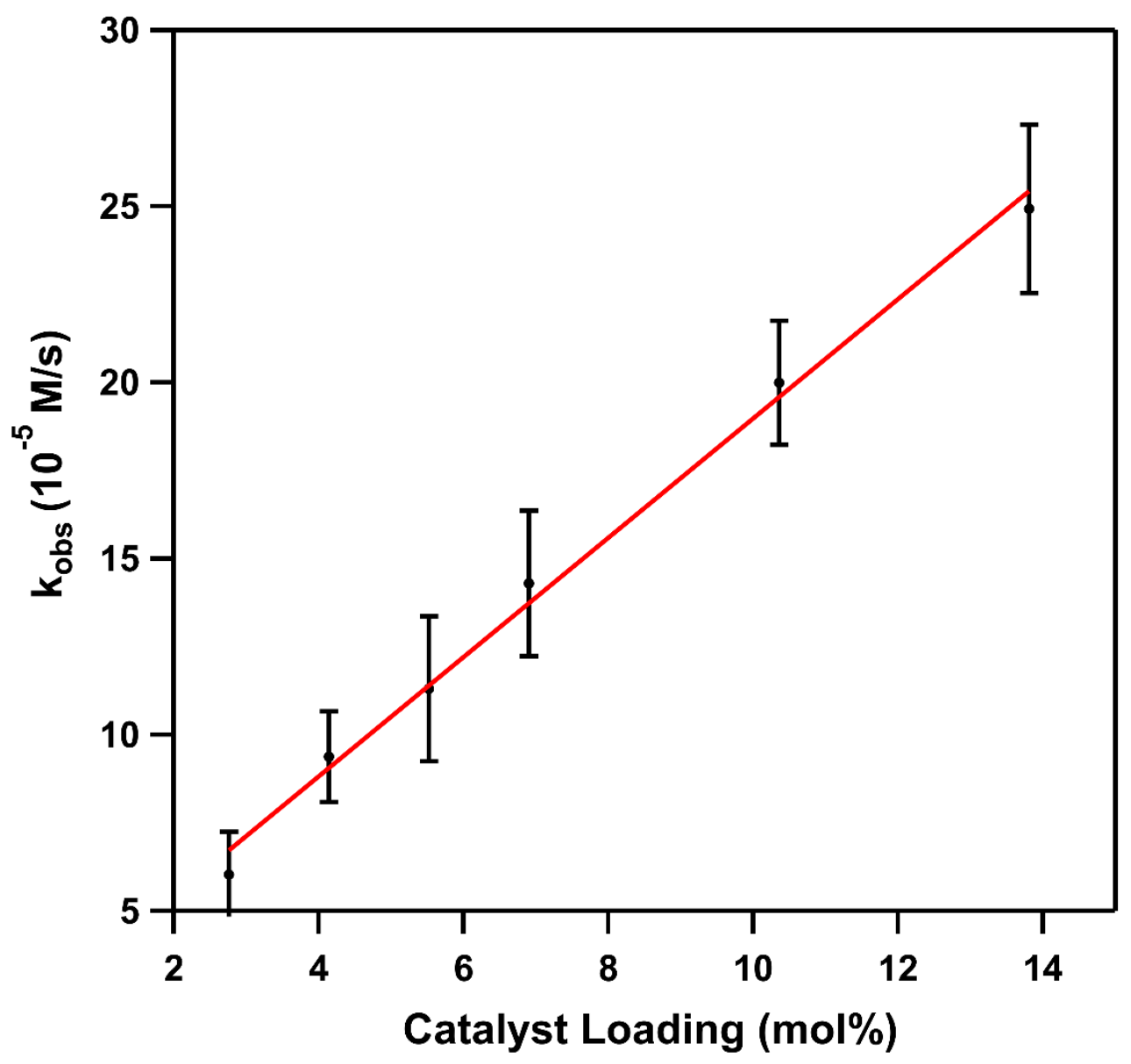

Linear relationship between observed rate constant $k_{\text {obs }}$ versus catalyst loadings (mol\%) suggests a first-order reaction rate dependence on catalyst concentration. 
Figure S3. Molecular Orbital Analysis for Reactivity Discrepancy in Benzene with or without the presence of $\mathrm{CH}_{3} \mathrm{CN}$

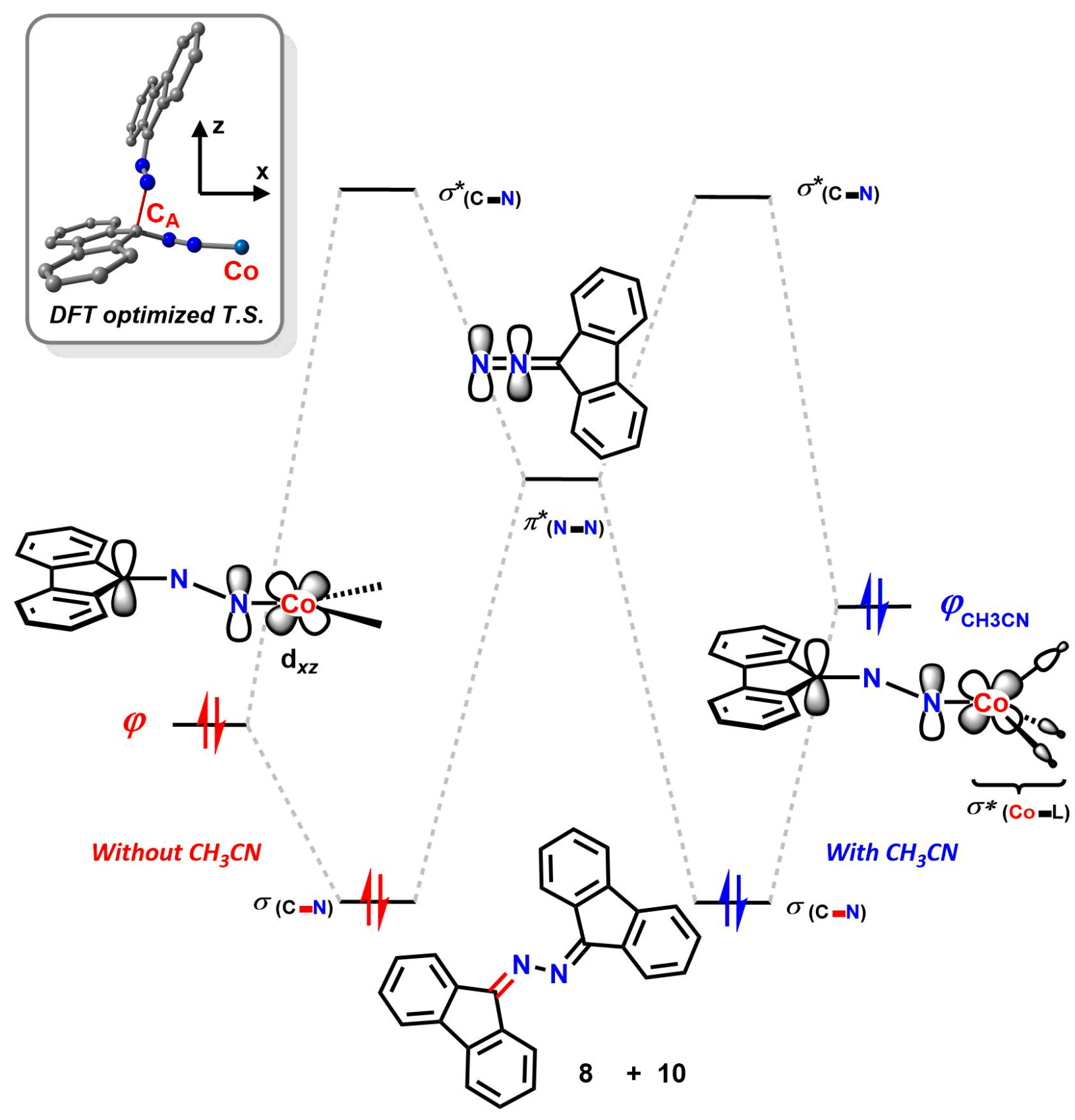

$\varphi_{\text {СнзсN }}$ is higher in energy than $\varphi$ due to the additional $\sigma^{*}$ character with spectator ligands, creating a better energy match with the electrophilic $\pi^{*}$ orbital of exogenous FluN2. 


\section{X-ray Diffraction Techniques}

Structures of 2-3 were collected at the UC Berkeley CHEXRAY crystallographic facility on a Bruker three-circle platform goniometer equipped with an Apex II CCD and an Oxford cryostream cooling device. Radiation was from a graphite fine-focus sealed tube $\mathrm{Mo} \mathrm{K} \alpha(0.71073$ $\AA$ A) source. Crystals were mounted on a cryoloop or glass fiber pin using Paratone N oil. Structures were collected at $100 \mathrm{~K}$. Data were collected as a series of $\varphi$ and/or $\omega$ scans.

Data were integrated using SAINT and scaled with multi-scan absorption correction using SADABS. ${ }^{3-4}$ The structures were solved by intrinsic phasing, direct methods, or Patterson maps using SHELXS-2014 and refined against $F^{2}$ on all data by full-matrix least-squares with SHELXL2014 with OLEX2 interface. ${ }^{5-7}$ All non-H atoms, including the disorder fragments, were located in different Fourier maps and then refined anisotropically. The restraints on bond lengths and constraints of the atomic displacement parameters on each pair of disorder fragments (SADI/SAME and EADP instructions of SHELXL-2014) $)^{6-7}$ as well as the restraints of the atomic displacement parameters (SIMU/RIGU instructions of SHELXL-2014) ${ }^{8}$ if necessary, have been applied for the disorder refinement. Hydrogen atoms were placed at idealized positions and refined using a riding model. The isotropic displacement parameters of all hydrogen atoms were constrained to be 1.2 times the parameters of the atoms they were linked to (1.5 times for methyl groups).

(IPr)Co[N(SiMe3)DIPP] (2): (CCDC: 2062020) The structure was solved in the monoclinic space group $P 21 / \mathrm{n}$ with four molecules per unit cell and one molecule in the asymmetric unit.

(IPr)Co(FluN 2$)$ [N(SiMe3)DIPP] (3): (CCDC: 2062021) The structure was solved in the monoclinic space group $P 21 / n$ with four molecules per unit cell and one molecule in the asymmetric unit. Five isopropyl groups, as well as the fluorenyl fragment, display positional Page S23 
disorder and were refined using restraints and constraints commands. There is also residue electron density resulted from a disordered solvent (pentane) molecule. Due to the refinement of several other disordered substituents, the solvent residue electron density is masked using the Olex2 solvent mask protocol. 
Table S1. X-ray Diffraction Experimental Details

\begin{tabular}{|c|c|c|}
\hline & (IPr)Co[N(SiMes)DIPP] (2) & $(\operatorname{IPr}) \operatorname{Co}\left(\mathrm{FluN}_{2}\right)\left[\mathrm{N}\left(\mathrm{SiMe}_{3}\right) \mathrm{DIPP}\right](3)$ \\
\hline Moiety Formula & $\mathrm{C}_{42} \mathrm{H}_{62} \mathrm{CoN}_{3} \mathrm{Si}$ & $\mathrm{C}_{55} \mathrm{H}_{70} \mathrm{CoN}_{5} \mathrm{Si}$ \\
\hline FW & 695.96 & 888.18 \\
\hline Crystal System & Monoclinic & Monoclinic \\
\hline Space Group (Z) & $\mathrm{P} 21 / \mathrm{n}$ & $\mathrm{P} 2{ }_{1} / \mathrm{n}$ \\
\hline $\mathbf{a}(\AA)$ & $11.0037(15)$ & $14.6645(6)$ \\
\hline b $(\AA)$ & $34.538(4)$ & $17.0613(7)$ \\
\hline c $(\AA)$ & $11.5723(15)$ & 22.1103(9) \\
\hline$\alpha\left({ }^{\circ}\right)$ & 90 & 90 \\
\hline$\beta\left({ }^{\circ}\right)$ & $113.990(6)$ & $107.908(2)$ \\
\hline$\gamma\left({ }^{\circ}\right)$ & 90 & 90 \\
\hline Volume $\left(\AA^{3}\right)$ & 4018.1(9) & $5263.9(4)$ \\
\hline Calc. $\rho\left(\mathrm{mg} / \mathrm{m}^{3}\right)$ & 1.151 & 1.121 \\
\hline$\mu\left(\mathrm{cm}^{-1}\right)$ & 3.853 & 0.387 \\
\hline Crystal size (mm) & $0.09 \times 0.07 \times 0.03$ & $0.15 \times 0.12 \times 0.11$ \\
\hline Reflections & 7073 & 9618 \\
\hline Completeness (to 20) & 0.989 & 0.992 \\
\hline GOF on $\mathrm{F}^{2}$ & 1.066 & 1.026 \\
\hline $\mathbf{R}_{1}, w_{\mathbf{R}_{2}}^{\mathrm{c}}[\mathbf{I}>\mathbf{2 \sigma ( I ) ]}$ & $0.0477,0.1193$ & $0.1055,0.2637$ \\
\hline
\end{tabular}




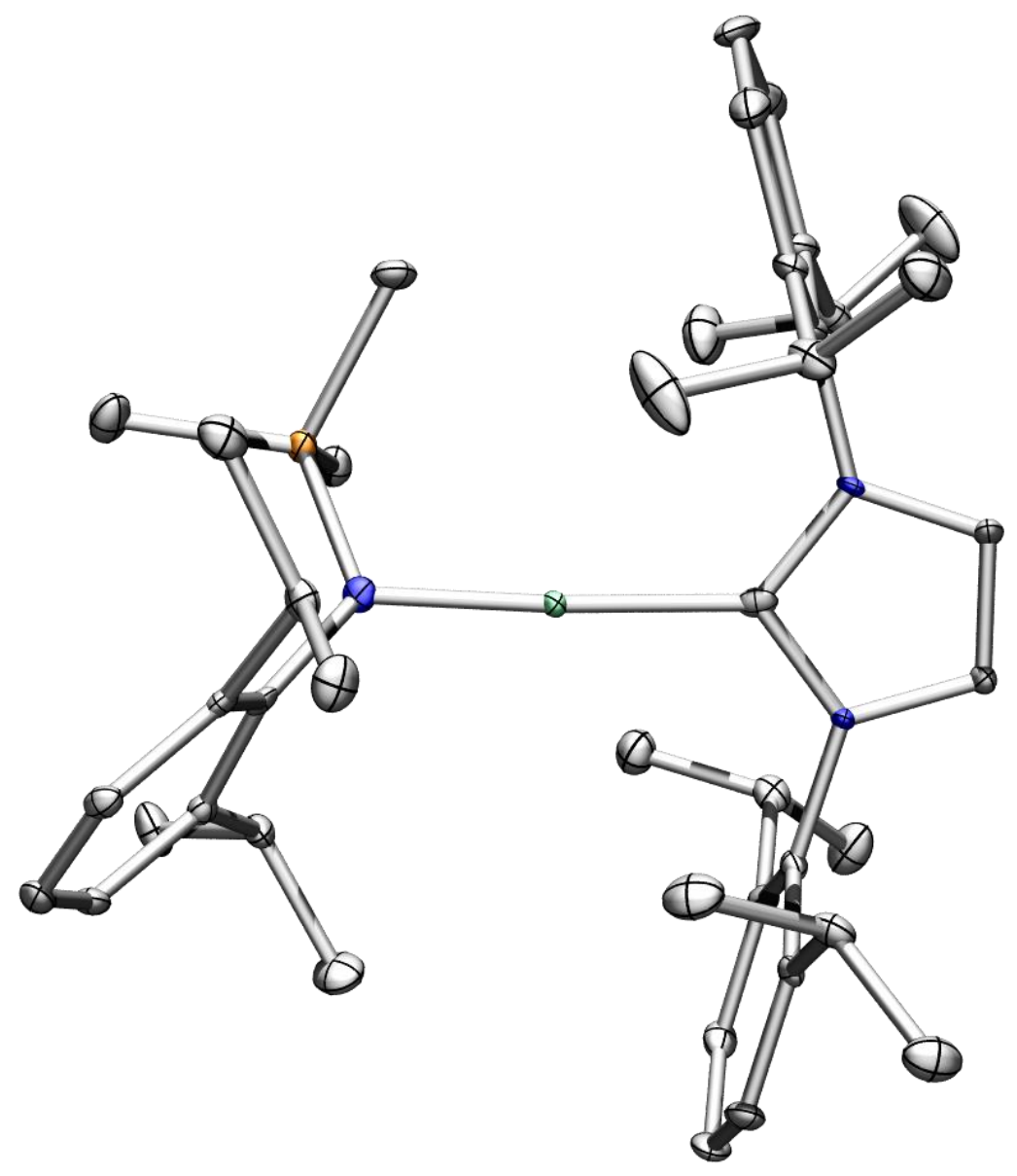

Figure S4. Solid-state structure of (IPr)Co[N(SiMe3)DIPP] (2) with thermal ellipsoids at 50\% probability level. Hydrogen atoms omitted for clarity . cobalt $=$ turquoise, nitrogen $=$ blue , carbon $=$ gray, silicon $=$ orange . 


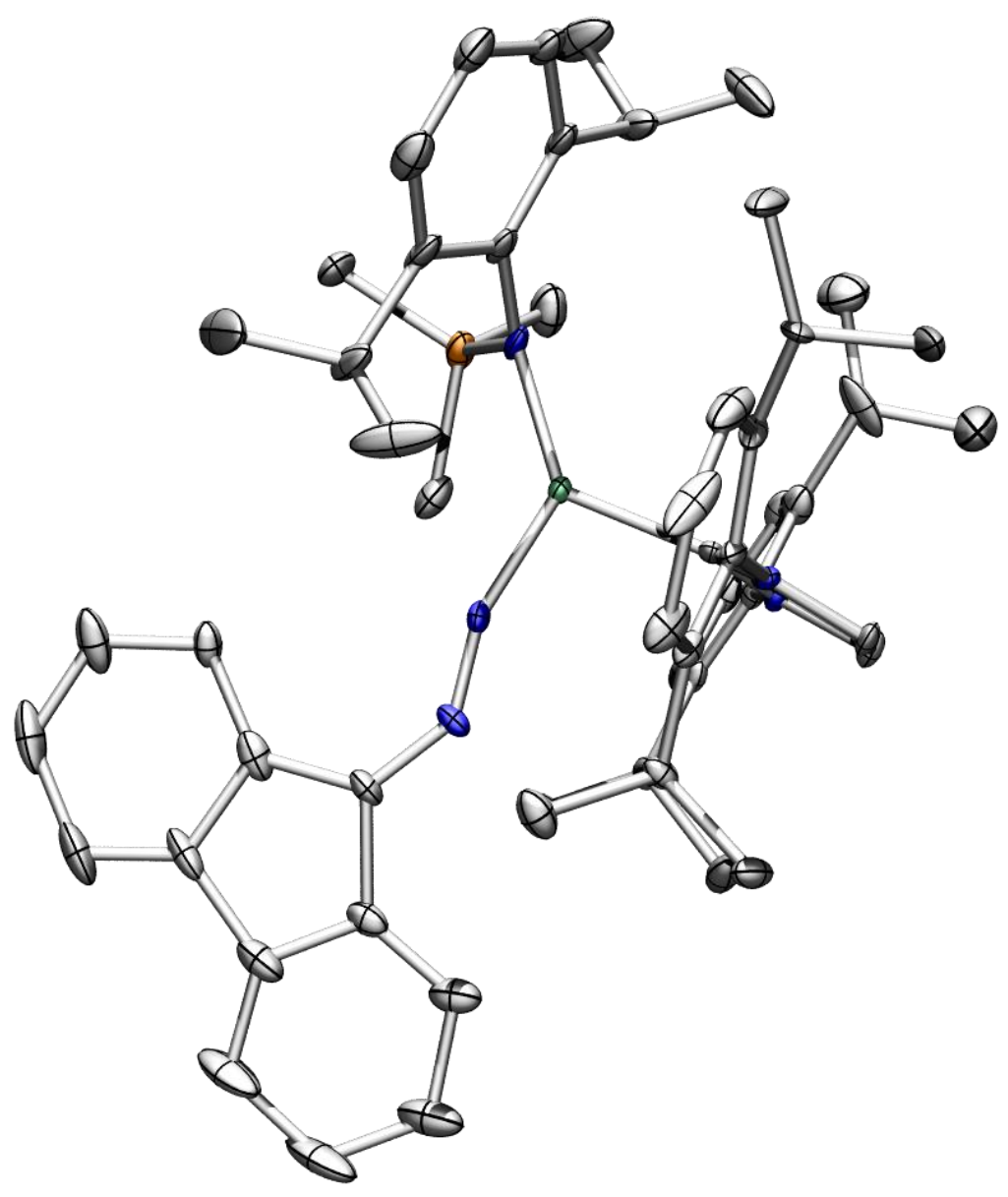

Figure S5. Solid-state structure of (IPr)Co(FluN 2$)\left[N\left(\mathrm{SiMe}_{3}\right) \mathrm{DIPP}\right](3)$ with thermal ellipsoids at $30 \%$ probability level. Hydrogen atoms omitted for clarity. cobalt $=$ turquoise, nitrogen $=$ blue, carbon $=$ gray, silicon $=$ orange. 


\section{Computational Methods}

Density functional theory (DFT) calculations were carried out with Gaussian 09 (Revision E.01). ${ }^{9}$ Geometry optimizations were performed at the unrestricted $\omega$ B97X-D level of theory. ${ }^{10}$ The Co and Si centers were described with Stuttgart RECPs and associated basis sets with added dpolarization on $\mathrm{Si}(\zeta=0.284) .{ }^{11-12}$ The $6-31 \mathrm{G}^{* *}$ basis set was used for all other atoms (denoted BS1). ${ }^{13-14}$ Additional single-point calculations were performed in benzene $(\varepsilon=2.2706)$ and THF $(\varepsilon=7.4257)$ through the solvation model based on density (SMD) approach. ${ }^{15}$ In this case, the Stuttgart RECPs and associated basis sets for Co and $\mathrm{Si}$ with added f-polarization $(\zeta=2.780)$ on Co and d-polarization on $\operatorname{Si}(\zeta=0.284)$ were employed, ${ }^{11-12,16}$ while the $6-311 \mathrm{G}^{* *}$ basis set was used for all other atoms. ${ }^{13-14,17}$ Stability analyses were performed in addition to analytical frequency calculations on all stationary points to ensure that geometries correspond to local minima (all positive eigenvalues) or transition state (one negative eigenvalue). IRC calculations and subsequent geometry optimizations were used to confirm the minima linked by each transition state. ${ }^{18,19}$ All reported energies are corrected for zero-point vibrational energy, while free energies (quoted at $298.15 \mathrm{~K}$ and $1 \mathrm{~atm}$ ) are corrected using the modified harmonic oscillator approximation proposed by Grimme free-rotor approximation was employed to treat low-lying vibrational modes. ${ }^{20}$

Finally, the energy span model proposed by Kozuch and co-workers was used: (i) to identify the TOF determining transition state (TDTS) and TOF determining intermediate (TDI), as well as (ii) to calculate the turnover frequencies (TOFs) by employing the AUTOF program. ${ }^{21-22}$

\section{Page S28}




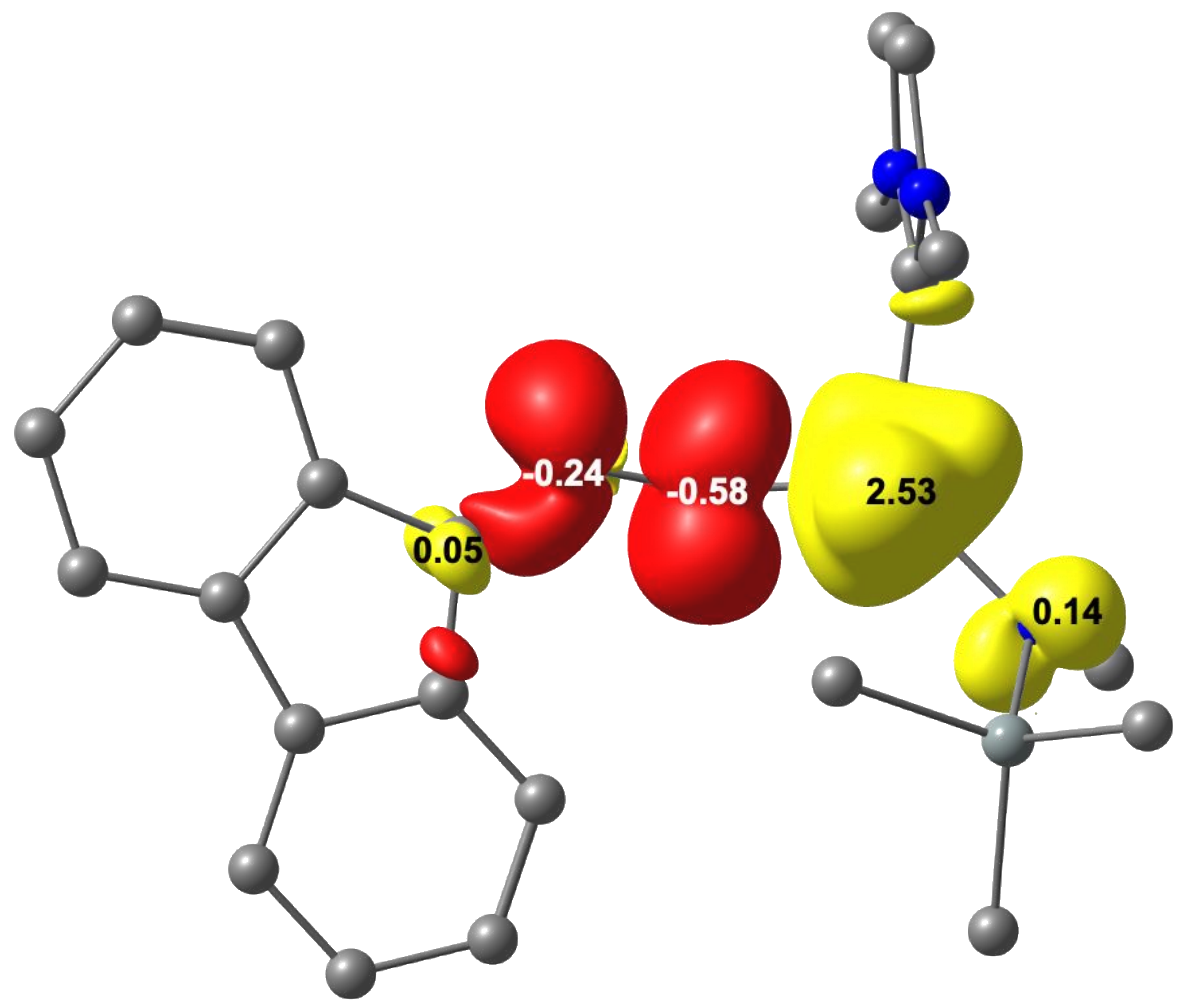

Figure S6. Isosurface $(0.004$ au) plot of the Mulliken spin population for $3(S=1)$. The diisopropylphenyl groups are truncated at the ipso carbon, and the hydrogen atoms are omitted for clarity. The Mulliken spin density plot indicates that this complex is best described as a high-spin divalent cobalt center $(S=3 / 2)$ antiferromagnetically coupled to a ligand-based radical $(S=-1 / 2)$, yielding an overall triplet spin ground state $(S=1)$. 


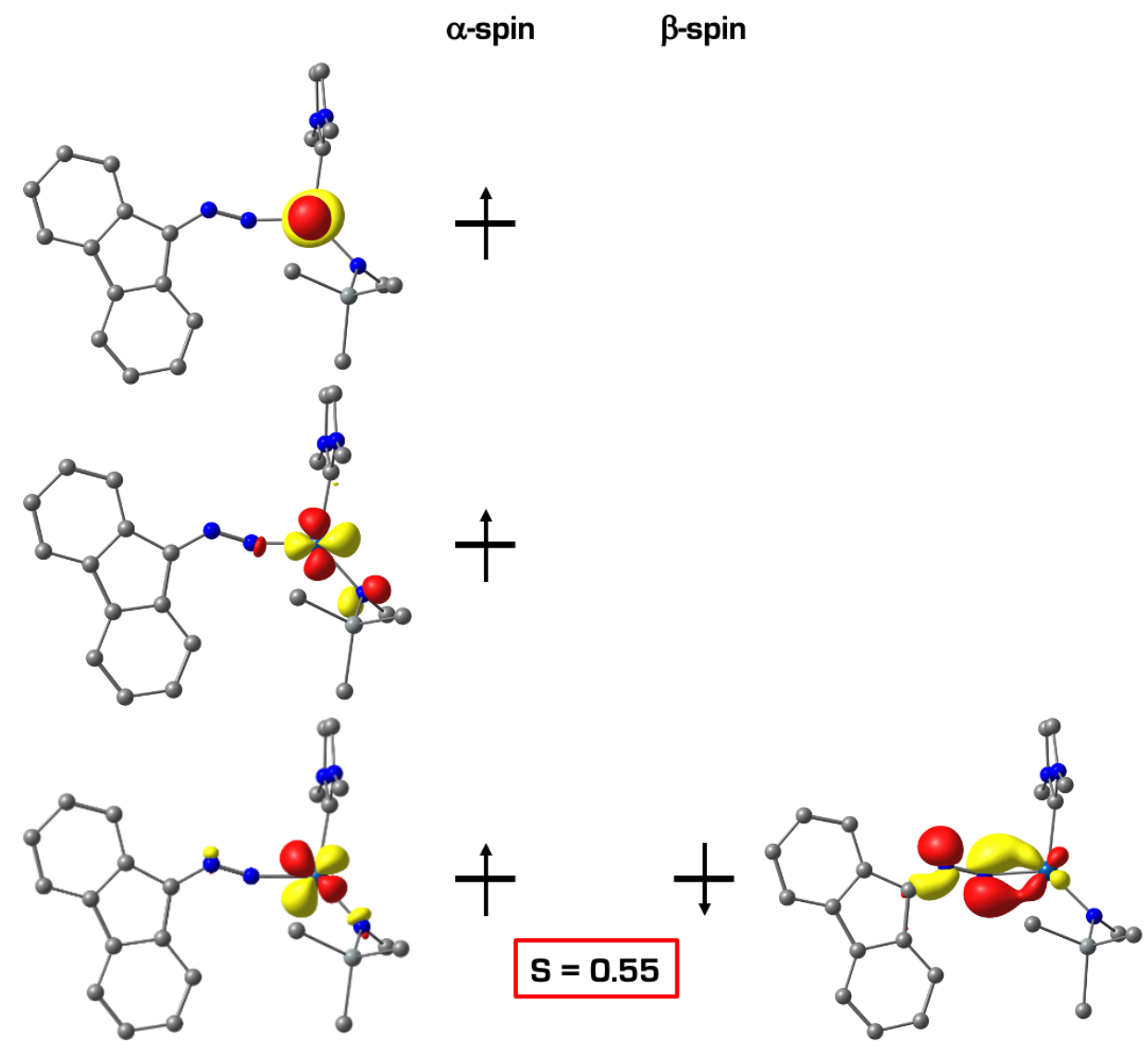

Figure S7. Isosurface $(0.004$ au $)$ plots of the maximally paired electrons for the a and $\beta$ frontier molecular orbitals (FMOs) in $3(\mathbf{S}=\mathbf{1})$ at the $\omega$ B97X-D/BS1 level of theory. The diisopropylphenyl groups are truncated at the ipso carbon, and the hydrogen atoms are omitted for clarity. The overlap integral is symbolized by $\mathrm{S}$. The value of $\mathrm{S}=0.55$ is similar to the diazoalkane adduct reported by Holland et al., which also features the same electronic structure description. ${ }^{23-}$ 24 

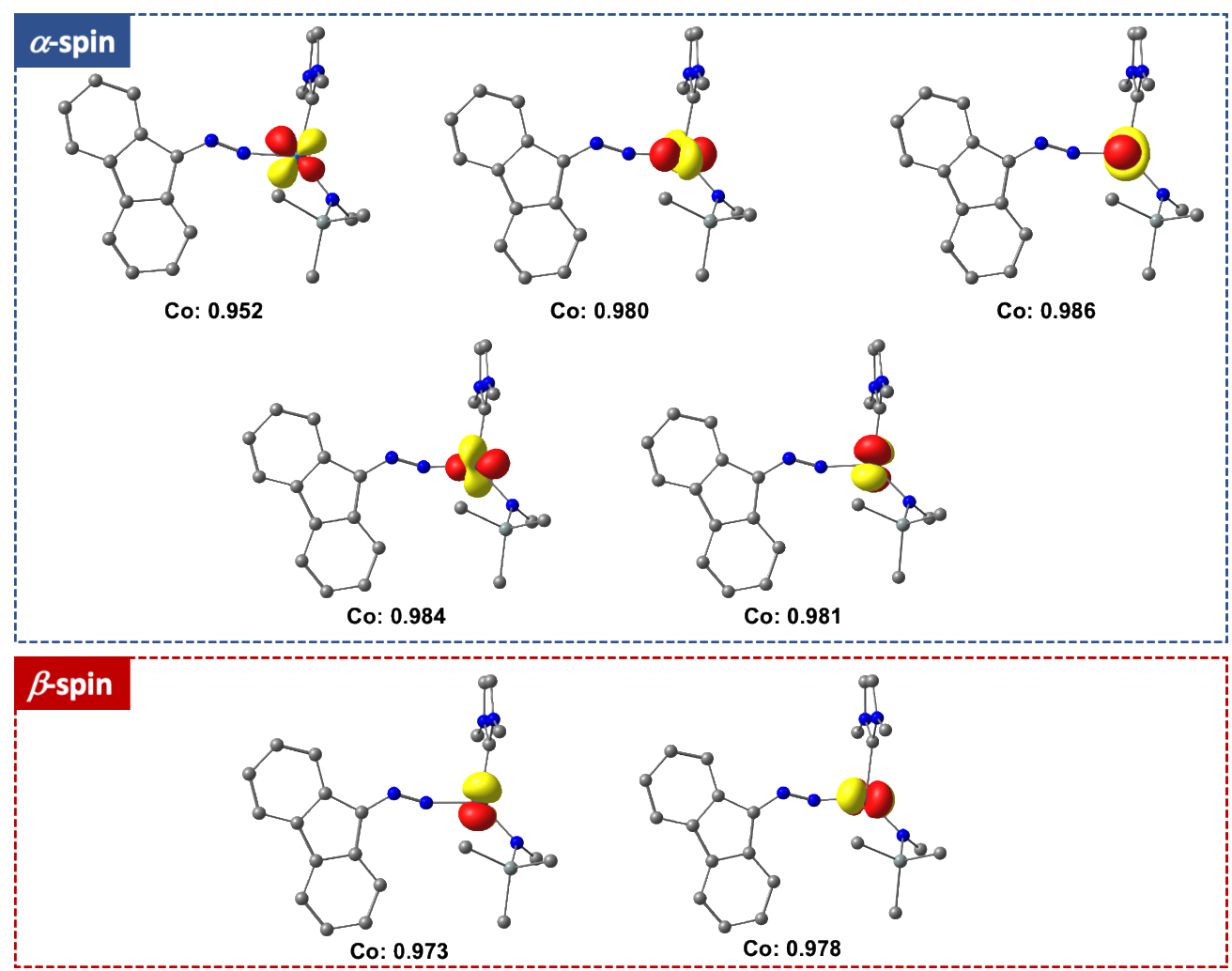

Figure S8. Isosurface (0.07 au) plots of the Pipek-Mezey localized d-electrons ( $\alpha$ - and $\beta$-spins) for $3\left(\mathbf{S}=\mathbf{1},\left\langle\mathbf{S}^{2}\right\rangle=\mathbf{2 . 7 5}\right)$. The Löwdin population analyses are provided for cobalt. The diisopropylphenyl groups are truncated at the ipso carbon, and the hydrogen atoms are omitted for clarity. LOBA analysis suggests that the oxidation state of cobalt is $+2\left(\mathrm{~d}^{7}\right)$.

In addition to plotting the Mulliken spin population (Figure S6) and the maximally paired electrons for the $a$ and $\beta$ molecular orbitals in 3 (Figure S7), the oxidation state of this species was established through localized orbital bonding analysis (LOBA) calculations on the $\omega \mathrm{B} 97 \mathrm{X}-\mathrm{D} / \mathrm{BS} 1$ optimized geometries, ${ }^{25}$ as implemented in the Q-Chem software. ${ }^{26}$ To avoid linear dependencies, the def2-SVP basis sets were used. In this case, the Pipek-Mezey orbitals were utilized in combination with the Löwdin population analysis. Our results indicate that the cobalt center is in a formal +2 oxidation state (Figure S8), consistent with our previous analysis.

\section{Page S31}


Figure S9. DFT calculated pathway for converting $\mathrm{FluN}_{2}$ to 9 in the presence of $\mathrm{CH}_{3} \mathrm{CN}$

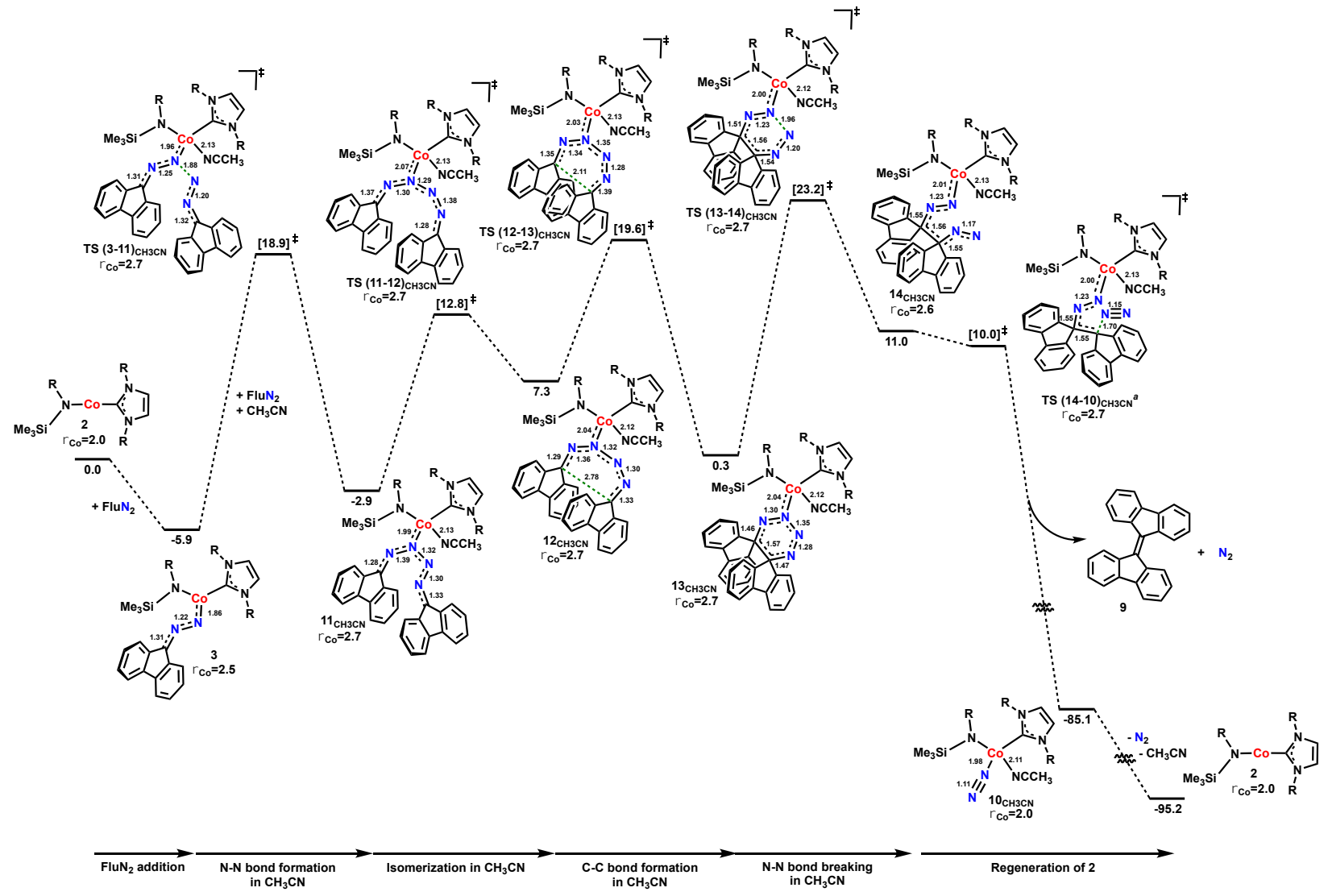

Computed Gibbs free energy ( $\Delta G, \mathrm{kcal} / \mathrm{mol})$ profile for the formation of 9,9'-bifluorenylidene (9) in the presence of $2, \mathrm{FluN}_{2}$, and $\mathrm{CH}_{3} \mathrm{CN}$. All free energies are calculated with respect to the separated reactants, and all complexes have a triplet ground-state. The spin on cobalt is denoted as $\rho_{\mathrm{Co}}$, and key distances are in angstroms. ${ }^{a}$ The forward reaction appears to be barrierless as the

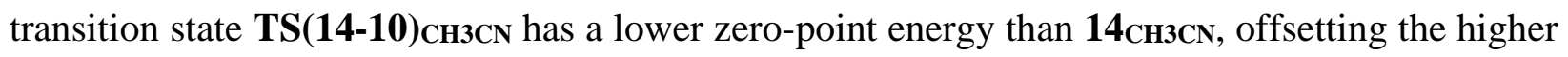
potential energy of the transition state with respect to the intermediate. 
Figure S10. Transition state for the formation of 8 in the presence of $\mathrm{CH}_{3} \mathrm{CN}$.

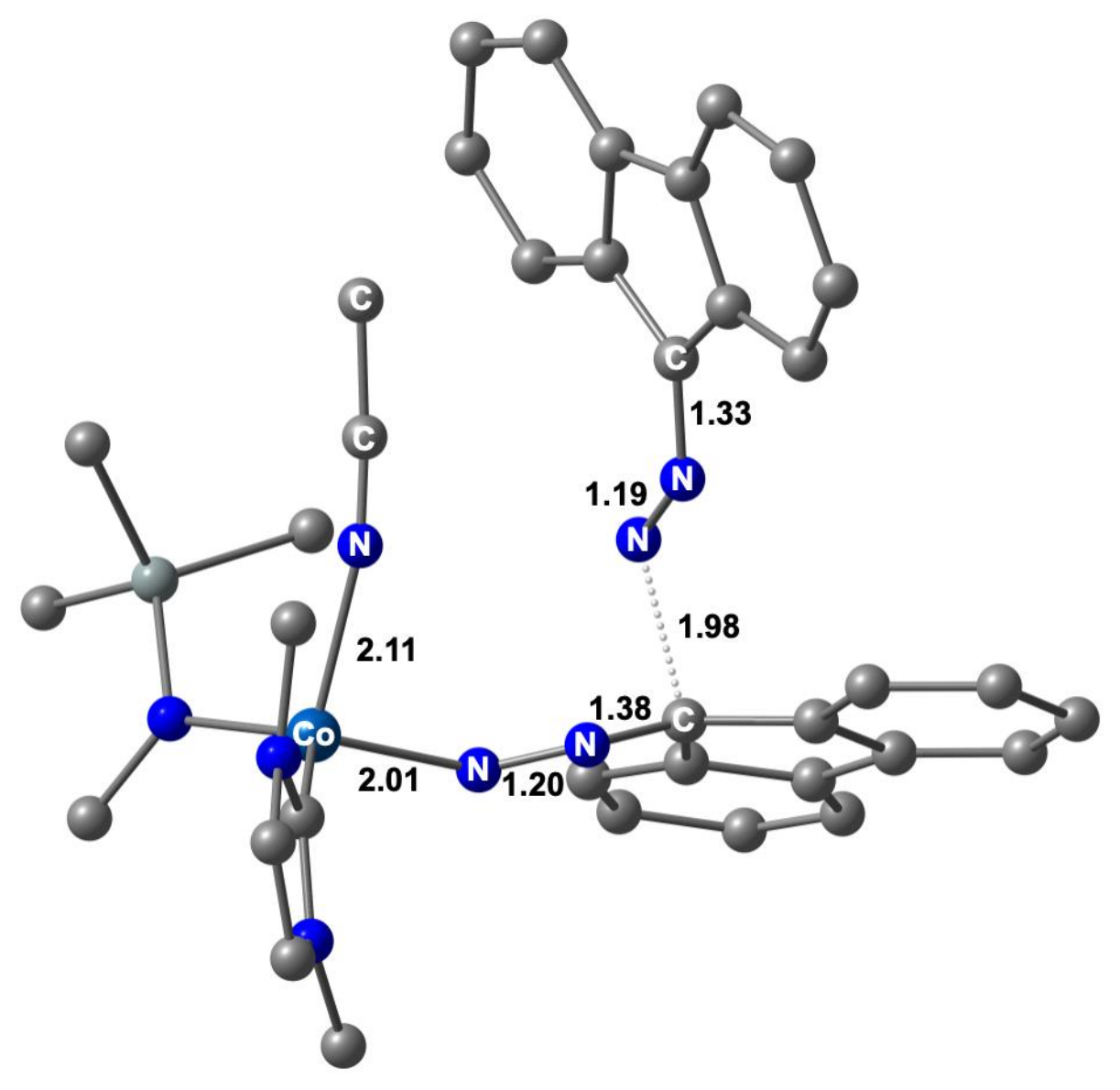

Computed transition state for the formation of 1,2-di(9H-fluoren-9-ylidene)hydrazine (8) in the presence of 2, FluN 2 , and $\mathrm{CH}_{3} \mathrm{CN}$. Key distances are in angstroms ( $\AA$ ). The diisopropylphenyl groups are truncated at the ipso carbon, and the hydrogen atoms are omitted for clarity. 


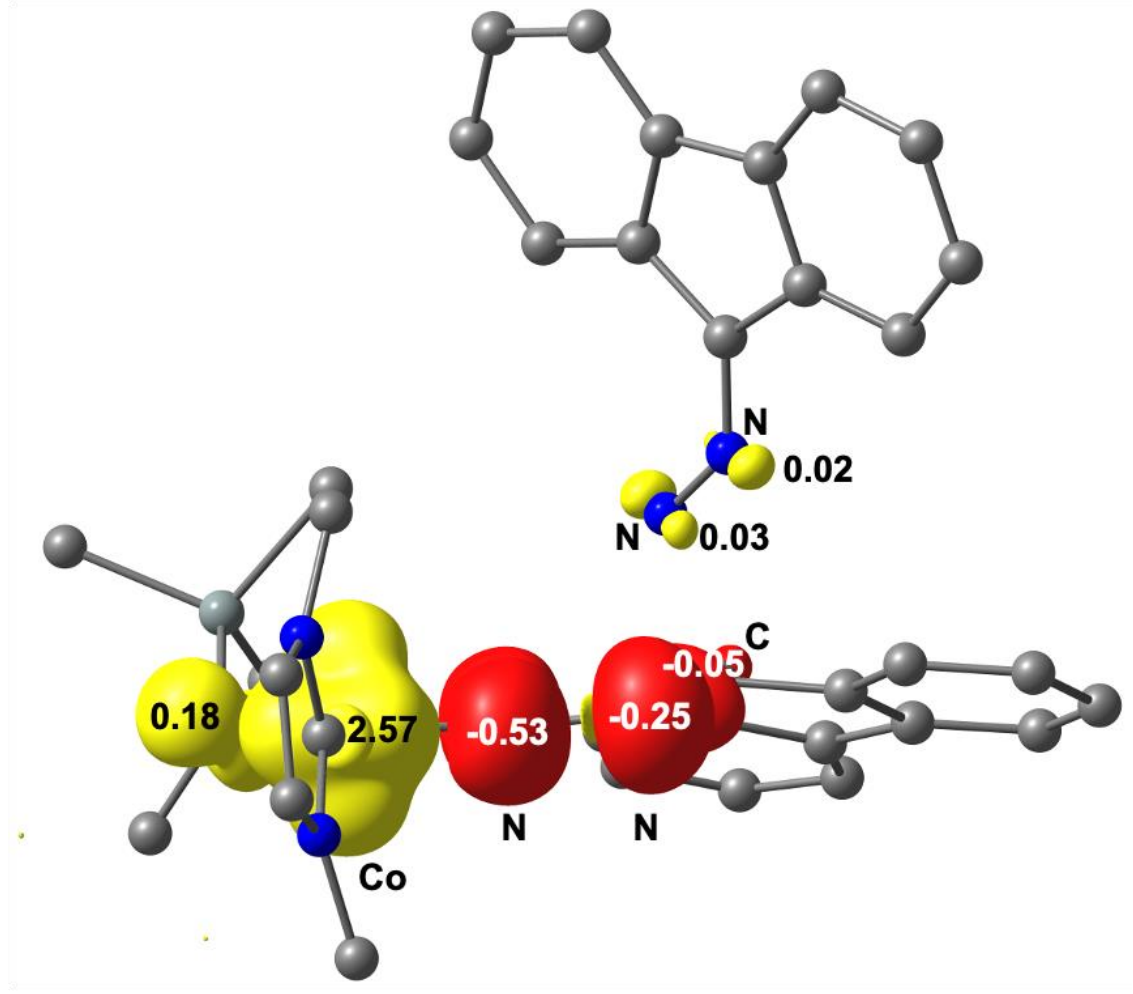

Figure S11. Isosurface (0.004 au) plot of the Mulliken spin population for TS $(3-10)(S=1)$.

The diisopropylphenyl groups are truncated at the ipso carbon, and the hydrogen atoms are omitted for clarity. 


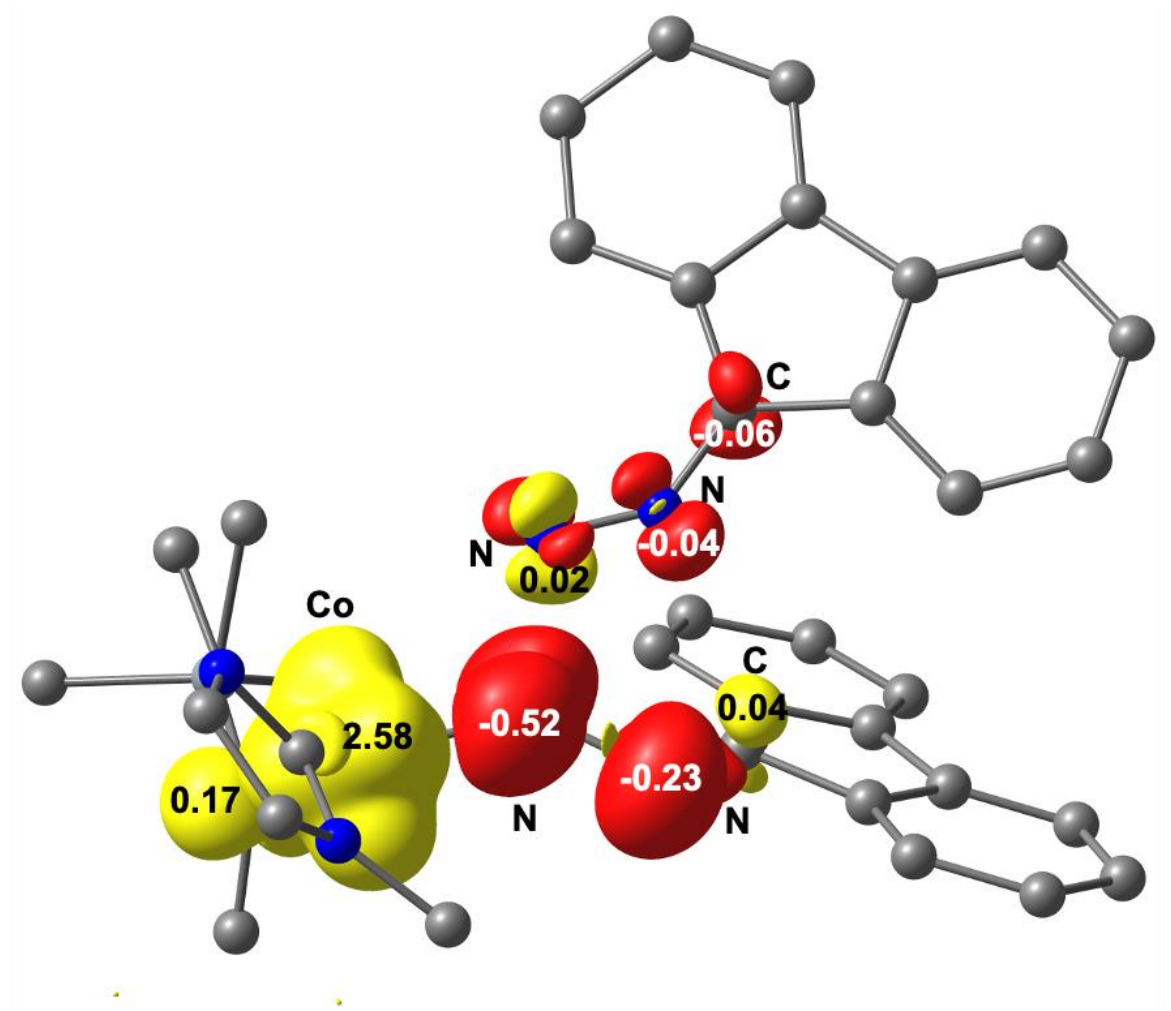

Figure S12. Isosurface (0.004 au) plot of the Mulliken spin population for TS(3-11) $(S=1)$.

The diisopropylphenyl groups are truncated at the ipso carbon, and the hydrogen atoms are omitted for clarity. 


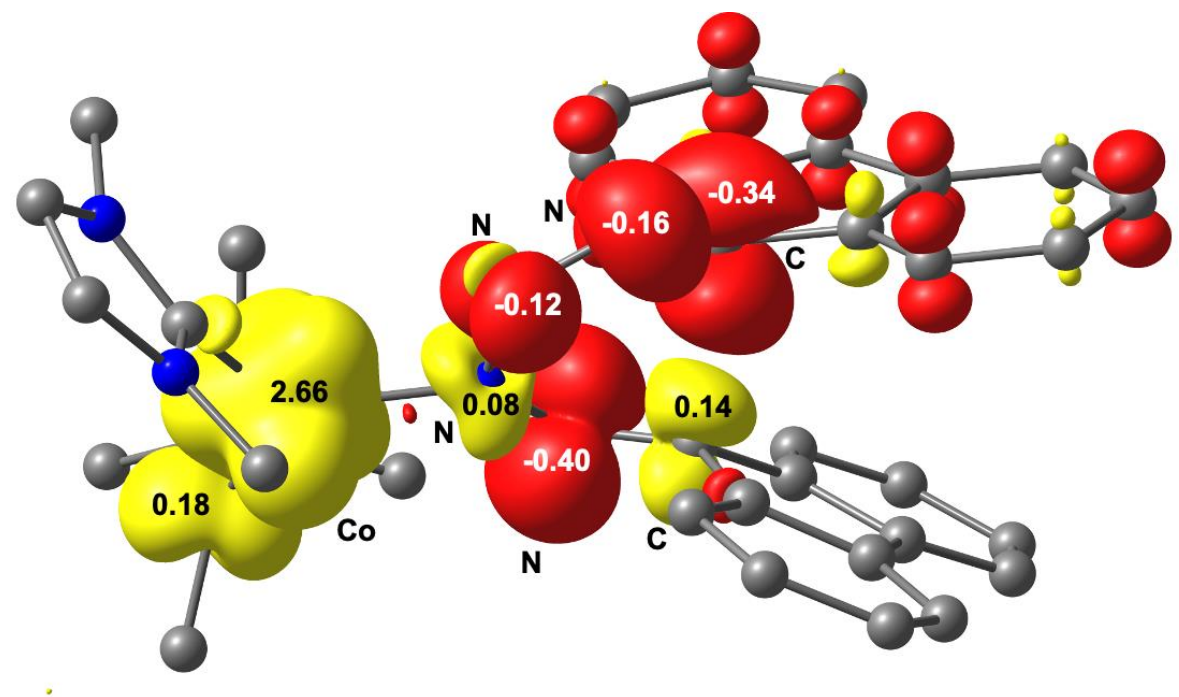

Figure S13. Isosurface (0.004 au) plot of the Mulliken spin population for $\operatorname{TS}(12-13)(S=1)$. The diisopropylphenyl groups are truncated at the ipso carbon, and the hydrogen atoms are omitted for clarity.

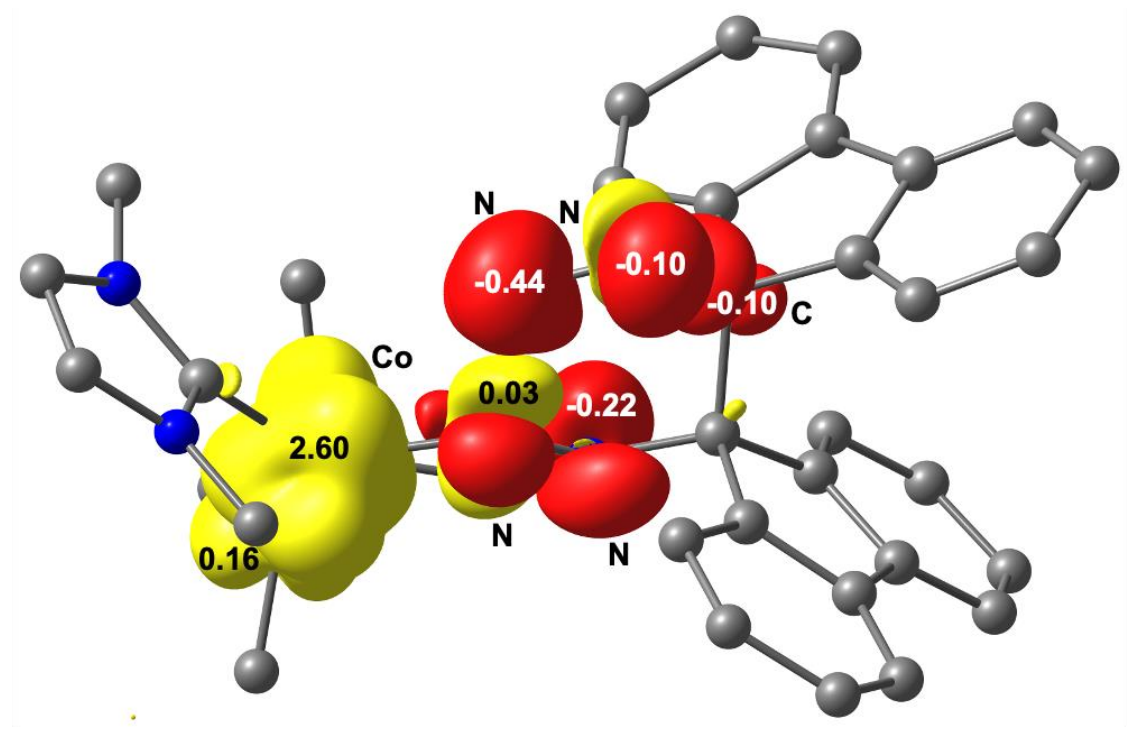

Figure S14. Isosurface (0.004 au) plot of the Mulliken spin population for TS(13-14) $(S=1)$. The diisopropylphenyl groups are truncated at the ipso carbon, and the hydrogen atoms are omitted for clarity. 
Table S2. Steps considered to calculate the TOF and $X_{T O F}$ for the formation of 8 in the presence of 2 and FluN 2 . The TOF is estimated to be $4.6 \times 10^{-7} \mathrm{~s}^{-1}$ at the $\omega \mathrm{B} 97 \mathrm{X}-$ $\mathrm{D}(\mathrm{SMD}) / \mathrm{BS} 2 / / \omega \mathrm{B} 97 \mathrm{X}-\mathrm{BS} 1$ level of theory. The "degree of TOF control" ( $\left.\mathrm{X}_{\mathrm{TOF}}\right)$ for the intermediates and transition states are given in Table S3. The overall change in the Gibbs free energy is $-59.2 \mathrm{kcal} / \mathrm{mol}$. The TOF determining intermediate (TDI) and the TOF determining transition state (TDTS) based on the energy span model are highlighted in gray.

\begin{tabular}{lcccc}
\hline & $\begin{array}{c}\text { Intermediates } \\
(\mathbf{k c a l} / \mathbf{m o l})\end{array}$ & \multicolumn{2}{c}{$\begin{array}{c}\text { Transition States } \\
(\mathbf{k c a l} / \mathbf{m o l})\end{array}$} \\
\hline Step 1 & 0.0 & $\mathbf{2}$ & 0.0 & \\
Step 2 & -5.9 & $\mathbf{3}$ & 20.2 & TS (3-10) \\
Step 3 & -51.9 & $\mathbf{1 0}$ & -51.9 & \\
\hline
\end{tabular}

Table S3. ХтоF of each intermediate and transition state for the formation of 8 in the presence of 2 and FluN2. The "degree of TOF control" for the TOF determining intermediate (TDI) and the TOF determining transition state (TDTS) based on the energy span model are highlighted in gray.

\begin{tabular}{lcccc}
\hline & & $\mathbf{X}_{\text {TOF, }} \boldsymbol{I}_{j}$ & \multicolumn{2}{c}{$\mathbf{X}_{\text {TOF, }} \boldsymbol{T}_{i}$} \\
\hline Step 1 & 0.00 & $\mathbf{2}$ & 0.00 & \\
Step 2 & 1.00 & $\mathbf{3}$ & 1.00 & TS (3-10) \\
Step 3 & 0.00 & $\mathbf{1 0}$ & 0.00 & \\
\hline
\end{tabular}


Table S4. Steps considered to calculate the TOF and $X_{\text {TOF }}$ for the formation of 9 in the presence of 2 and FluN 2 . The TOF is estimated to be $2.3 \times 10^{-6} \mathrm{~s}^{-1}$ at the $\omega \mathrm{B} 97 \mathrm{X}-$ $\mathrm{D}(\mathrm{SMD}) / \mathrm{BS} 2 / / \omega \mathrm{B} 97 \mathrm{X}-\mathrm{BS} 1$ level of theory. The "degree of TOF control" ( $\left.\mathrm{X}_{\mathrm{TOF}}\right)$ for the intermediates and transition states are given in Table S5. ${ }^{\mathrm{a}}$ The Gibbs free energy of the highest intermediate was employed for this transition state. The overall change in the Gibbs free energy is $-95.2 \mathrm{kcal} / \mathrm{mol}$. The TOF determining intermediate (TDI) and the TOF determining transition state (TDTS) based on the energy span model are highlighted in gray.

\begin{tabular}{lcccc}
\hline & \multicolumn{2}{c}{$\begin{array}{c}\text { Intermediates } \\
(\mathrm{kcal} / \mathbf{m o l})\end{array}$} & \multicolumn{2}{c}{$\begin{array}{c}\text { Transition States } \\
(\mathrm{kcal} / \mathbf{m o l})\end{array}$} \\
\hline Step 1 & 0.0 & $\mathbf{2}$ & 0.0 & \\
Step 2 & -5.9 & $\mathbf{3}$ & 16.4 & TS (3-11) \\
Step 3 & -8.1 & $\mathbf{1 1}$ & 10.6 & TS (11-12) \\
Step 4 & 4.1 & $\mathbf{1 2}$ & 16.0 & TS (12-13) \\
Step 5 & -3.4 & $\mathbf{1 3}$ & 16.9 & TS (13-14) \\
Step 6 & 5.3 & $\mathbf{1 4}$ & $5.3^{a}$ & TS (14-10) \\
Step 7 & -87.9 & $\mathbf{1 0}$ & -87.9 & \\
\hline
\end{tabular}


Table S5. $\mathrm{X}_{\mathrm{TOF}}$ of each intermediate and transition state for the formation of $9 \mathrm{in}$ the presence of $\mathbf{2}$ and FluN2. The "degree of TOF control" for the TOF determining intermediate (TDI) and the TOF determining transition state (TDTS) based on the energy span model are highlighted in gray.

\begin{tabular}{rrrrr}
\hline & \multicolumn{2}{c}{$\mathrm{X}_{\text {TOF }}, I_{j}$} & \multicolumn{2}{c}{$\mathrm{X}_{\text {TOF, }} \boldsymbol{T}_{i}$} \\
\hline Step 1 & 0.00 & $\mathbf{2}$ & 0.00 & \\
Step 2 & 0.03 & $\mathbf{3}$ & 0.01 & TS (3-11) \\
Step 3 & 0.97 & $\mathbf{1 1}$ & 0.00 & TS (11-12) \\
Step 4 & 0.00 & $\mathbf{1 2}$ & 0.18 & TS (12-13) \\
Step 5 & 0.00 & $\mathbf{1 3}$ & 0.81 & TS (13-14) \\
Step 6 & 0.00 & $\mathbf{1 4}$ & 0.00 & TS (14-10) \\
Step 7 & 0.00 & $\mathbf{1 0}$ & 0.00 & \\
\hline
\end{tabular}


Table S6. Steps considered to calculate the TOF and $X_{\text {TOF }}$ for the formation of 8 in the presence of 2, FluN 2 and $\mathrm{CH}_{3} \mathrm{CN}$. Acetonitrile was employed to model tert-butylnitrile as the Ltype donor. The TOF is estimated to be $1.6 \times 10^{-5} \mathrm{~s}^{-1}$ at the $\omega \mathrm{B} 97 \mathrm{X}-\mathrm{D}(\mathrm{SMD}) / \mathrm{BS} 2 / / \omega \mathrm{B} 97 \mathrm{X}-\mathrm{BS} 1$ level of theory. The "degree of TOF control" $\left(\mathrm{X}_{\mathrm{TOF}}\right)$ for the intermediates and transition states are given in Table S7. The overall change in the Gibbs free energy is $-59.2 \mathrm{kcal} / \mathrm{mol}$. The TOF determining intermediate (TDI) and the TOF determining transition state (TDTS) based on the energy span model are highlighted in gray.

\begin{tabular}{ccccc}
\hline & \multicolumn{2}{c}{$\begin{array}{c}\text { Intermediates } \\
(\mathbf{k c a l} / \mathbf{m o l})\end{array}$} & \multicolumn{2}{c}{$\begin{array}{c}\text { Transition States } \\
(\mathbf{k c a l} / \mathbf{m o l})\end{array}$} \\
\hline Step 1 & 0.0 & $\mathbf{2}$ & 0.0 & \\
Step 2 & -5.9 & $\mathbf{3}$ & 18.1 & TS (3-10) CH3CN \\
Step 3 & -49.1 & $\mathbf{1 0}$ CH3CN & -49.1 & \\
\hline
\end{tabular}

Table S7. X of 2, FluN 2 and $\mathrm{CH}_{3} \mathrm{CN}$. Acetonitrile was employed to model tert-butylnitrile as the L-type donor. The "degree of TOF control" for the TOF determining intermediate (TDI) and the TOF determining transition state (TDTS) based on the energy span model are highlighted in gray.

\begin{tabular}{lcccc}
\hline & \multicolumn{3}{c}{$\mathbf{X}_{\text {TOF, }} \boldsymbol{I}_{j}$} & \multicolumn{2}{c}{$\mathbf{X}_{\text {TOF }}, \boldsymbol{T}_{i}$} \\
\hline Step 1 & 0.00 & $\mathbf{2}$ & 0.00 & \\
Step 2 & 1.00 & $\mathbf{3}$ & 1.00 & TS (3-10) CH3CN \\
Step 3 & 0.00 & $\mathbf{1 0}_{\mathrm{CH} 3 \mathrm{CN}}$ & 0.00 & \\
\hline
\end{tabular}


Table S8. Steps considered to calculate the TOF and $\mathrm{X}_{\mathrm{TOF}}$ for the formation of 9 in the presence of 2, FluN $N_{2}$ and $\mathrm{CH}_{3} \mathrm{CN}$. Acetonitrile was employed to model tert-butylnitrile as the Ltype donor. The TOF is estimated to be $2.9 \times 10^{-9} \mathrm{~s}^{-1}$ at the $\omega \mathrm{B} 97 \mathrm{X}-\mathrm{D}(\mathrm{SMD}) / \mathrm{BS} 2 / / \omega \mathrm{B} 97 \mathrm{X}-\mathrm{BS} 1$ level of theory. The "degree of TOF control" $\left(\mathrm{X}_{\mathrm{TOF}}\right)$ for the intermediates and transition states are given in Table S9. ${ }^{a}$ The Gibbs free energy of the highest intermediate was employed for this transition state. The overall change in the Gibbs free energy is $-95.2 \mathrm{kcal} / \mathrm{mol}$. The TOF determining intermediate (TDI) and the TOF determining transition state (TDTS) based on the energy span model are highlighted in gray.

\begin{tabular}{|c|c|c|c|c|}
\hline \multirow[b]{2}{*}{ Step 1} & \multicolumn{2}{|c|}{$\begin{array}{c}\text { Intermediates } \\
\text { (kcal/mol) }\end{array}$} & \multicolumn{2}{|c|}{$\begin{array}{c}\text { Transition States } \\
\text { (kcal/mol) }\end{array}$} \\
\hline & 0.0 & 2 & 0.0 & \\
\hline Step 2 & -5.9 & 3 & 18.9 & TS (3-11) CH3CN \\
\hline Step 3 & -2.9 & $11 \mathrm{CH} 3 \mathrm{CN}$ & 12.8 & $\mathrm{TS}(11-12)_{\mathrm{CH} 3 \mathrm{CN}}$ \\
\hline Step 4 & 7.3 & $12 \mathrm{CH} 3 \mathrm{CN}$ & 19.6 & TS $(12-13)_{\mathrm{CH}} \mathrm{CN}$ \\
\hline Step 5 & 0.3 & $13 \mathrm{CH} 3 \mathrm{CN}$ & 23.2 & TS $(13-14)_{\mathrm{CH}} \mathrm{CN}$ \\
\hline Step 6 & 11.0 & $14 \mathrm{CH} 3 \mathrm{CN}$ & $11.0^{a}$ & TS $(14-10) \mathrm{CH} 3 \mathrm{CN}$ \\
\hline Step 7 & -85.1 & $10 \mathrm{CH} 3 \mathrm{CN}$ & -85.1 & \\
\hline
\end{tabular}


Table S9. $\mathrm{X}_{\mathrm{TOF}}$ of each intermediate and transition state for the formation of 9 in the presence of 2, FluN 2 and $\mathrm{CH}_{3} \mathrm{CN}$. Acetonitrile was employed to model tert-butylnitrile as the L-type donor. The "degree of TOF control" for the TOF determining intermediate (TDI) and the TOF determining transition state (TDTS) based on the energy span model are highlighted in gray.

\begin{tabular}{|c|c|c|c|c|}
\hline & \multicolumn{2}{|c|}{$\overline{\mathbf{X}_{\mathrm{TOF}}, \boldsymbol{I}_{j}}$} & \multicolumn{2}{|c|}{$\overline{\mathbf{X}_{\text {TOF }}, T_{i}}$} \\
\hline Step 1 & 0.00 & 2 & 0.00 & \\
\hline Step 2 & 0.99 & 3 & 0.00 & TS $(3-11){ }_{\mathrm{CH} 3 \mathrm{CN}}$ \\
\hline Step 3 & 0.01 & $11 \mathrm{CH}_{\mathrm{CHN}}$ & 0.00 & TS $(11-12)_{\mathrm{CH} 3 \mathrm{CN}}$ \\
\hline Step 4 & 0.00 & $12 \mathrm{CH} 3 \mathrm{CN}$ & 0.00 & $\mathrm{TS}(12-13)_{\mathrm{CH} 3 \mathrm{CN}}$ \\
\hline Step 5 & 0.00 & $13 \mathrm{CH} 3 \mathrm{CN}$ & 1.00 & TS $(13-14)_{\mathrm{CH} 3 \mathrm{CN}}$ \\
\hline Step 6 & 0.00 & $14_{\mathrm{CH} 3 \mathrm{CN}}$ & 0.00 & TS $(14-10)_{\mathrm{CH}} \mathrm{CN}$ \\
\hline Step 7 & 0.00 & $10 \mathrm{CH} 3 \mathrm{CN}$ & 0.00 & \\
\hline
\end{tabular}

Table S10. Steps considered to calculate the TOF and $X_{\text {TOF }}$ for the formation of 8 in the presence of 2 and FluN $\mathbf{N}_{2}$ in THF. The TOF is estimated to be $2.6 \times 10^{-5} \mathrm{~s}^{-1}$ at the $\omega \mathrm{B} 97 \mathrm{X}$ $\mathrm{D}(\mathrm{SMD}) / \mathrm{BS} 2 / / \omega \mathrm{B} 97 \mathrm{X}-\mathrm{BS} 1$ level of theory. The "degree of TOF control" ( $\left.\mathrm{X}_{\mathrm{TOF}}\right)$ for the intermediates and transition states are given in Table S11. The overall change in the Gibbs free energy is $-58.7 \mathrm{kcal} / \mathrm{mol}$. The TOF determining intermediate (TDI) and the TOF determining transition state (TDTS) based on the energy span model are highlighted in gray.

\begin{tabular}{ccccc}
\hline & \multicolumn{2}{c}{$\begin{array}{c}\text { Intermediates } \\
(\mathbf{k c a l} / \mathbf{m o l})\end{array}$} & \multicolumn{2}{c}{$\begin{array}{c}\text { Transition States } \\
(\mathbf{k c a l} / \mathbf{m o l})\end{array}$} \\
\hline Step 1 & 0.0 & $\mathbf{2}$ & 0.0 & \\
Step 2 & -7.3 & $\mathbf{3}$ & 16.4 & TS (3-10) \\
Step 3 & -51.5 & $\mathbf{1 0}$ & -51.5 & \\
\hline
\end{tabular}


Table S11. $\mathrm{X}_{\text {тоF }}$ of each intermediate and transition state for the formation of 8 in the presence of 2 and FluN 2 in THF. The "degree of TOF control" for the TOF determining intermediate (TDI) and the TOF determining transition state (TDTS) based on the energy span model are highlighted in gray.

\begin{tabular}{lcccc}
\hline & \multicolumn{1}{c}{$\mathbf{X}_{\text {TOF, }} \boldsymbol{I}_{j}$} & \multicolumn{2}{c}{$\mathbf{X}_{\text {TOF, }} \boldsymbol{T}_{i}$} \\
\hline Step 1 & 0.00 & $\mathbf{2}$ & 0.00 & \\
Step 2 & 1.00 & $\mathbf{3}$ & 1.00 & TS (3-10) \\
Step 3 & 0.00 & $\mathbf{1 0}$ & 0.00 & \\
\hline
\end{tabular}

Table S12. Steps considered to calculate the TOF and $X_{\text {TOF }}$ for the formation of 9 in the presence of 2 and FluN 2 in THF. The TOF is estimated to be $6.4 \times 10^{-6} \mathrm{~s}^{-1}$ at the $\omega \mathrm{B} 97 \mathrm{X}-$ $\mathrm{D}(\mathrm{SMD}) / \mathrm{BS} 2 / / \omega \mathrm{B} 97 \mathrm{X}-\mathrm{BS} 1$ level of theory. The "degree of TOF control" ( $\left.\mathrm{X}_{\mathrm{TOF}}\right)$ for the

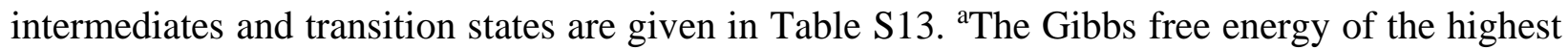
intermediate was employed for this transition state. The overall change in the Gibbs free energy is $-94.4 \mathrm{kcal} / \mathrm{mol}$. The TOF determining intermediate (TDI) and the TOF determining transition state (TDTS) based on the energy span model are highlighted in gray.

\begin{tabular}{ccccc}
\hline & \multicolumn{2}{c}{$\begin{array}{c}\text { Intermediates } \\
(\mathbf{k c a l} / \mathbf{m o l})\end{array}$} & \multicolumn{2}{c}{$\begin{array}{c}\text { Transition States } \\
(\mathbf{k c a l} / \mathbf{m o l})\end{array}$} \\
\hline Step 1 & 0.0 & $\mathbf{2}$ & 0.0 & \\
Step 2 & -7.3 & $\mathbf{3}$ & 13.9 & TS (3-11) \\
Step 3 & -10.4 & $\mathbf{1 1}$ & 7.6 & TS (11-12) \\
Step 4 & 1.1 & $\mathbf{1 2}$ & 13.2 & TS (12-13) \\
Step 5 & -6.3 & $\mathbf{1 3}$ & 14.0 & TS (13-14) \\
Step 6 & 2.3 & $\mathbf{1 4}$ & $2.3^{a}$ & TS (14-10) \\
Step 7 & -87.2 & $\mathbf{1 0}$ & -87.2 & \\
\hline
\end{tabular}


Table S13. $\mathrm{X}_{\text {тоF }}$ of each intermediate and transition state for the formation of 9 in the presence of 2 and FluN 2 in THF. The "degree of TOF control" for the TOF determining intermediate (TDI) and the TOF determining transition state (TDTS) based on the energy span model are highlighted in gray.

\begin{tabular}{lcccc}
\hline & & $\mathbf{X}_{\text {TOF }}, \boldsymbol{I}_{j}$ & \multicolumn{2}{c}{$\mathbf{X}_{\text {TOF, }} \boldsymbol{T}_{i}$} \\
\hline Step 1 & 0.00 & $\mathbf{2}$ & 0.00 & \\
Step 2 & 0.01 & $\mathbf{3}$ & 0.01 & TS (3-11) \\
Step 3 & 0.99 & $\mathbf{1 1}$ & 0.00 & TS (11-12) \\
Step 4 & 0.00 & $\mathbf{1 2}$ & 0.20 & TS (12-13) \\
Step 5 & 0.00 & $\mathbf{1 3}$ & 0.79 & TS (13-14) \\
Step 6 & 0.00 & $\mathbf{1 4}$ & 0.00 & TS (14-10) \\
Step 7 & 0.00 & $\mathbf{1 0}$ & 0.00 & \\
\hline
\end{tabular}

Table S14. Calculated relative energies for 2 at the $\omega \mathrm{B97X}-\mathrm{D} / \mathrm{BS2}(\mathrm{SMD}) / / \omega \mathrm{B} 97 \mathrm{X}-\mathrm{D} / \mathrm{BS} 1$ level of theory for different spin states. The Mulliken spin population is given for cobalt, $\rho_{\mathrm{Co}}$. The singlet-state species has a negative eigenvalue on the order of $57.1 \mathrm{~cm}^{-1}$.

\begin{tabular}{|c|c|c|c|c|c|}
\hline Multiplicity & $\left\langle\mathbf{S}^{\mathbf{2}}\right\rangle$ & $\boldsymbol{\rho}_{\mathrm{Co}}$ & $\Delta \mathbf{E}$ & $\Delta \mathbf{Z P E}$ & $\Delta \mathbf{G}$ \\
\hline $\mathrm{S}=0$ & 1.00 & -0.05 & 0.0 & 0.0 & 0.0 \\
\hline $\mathrm{S}=1$ & 2.02 & 1.98 & -18.8 & -18.7 & -20.0 \\
\hline $\mathrm{S}=2$ & 6.01 & 3.33 & 23.1 & 22.9 & 21.7 \\
\hline
\end{tabular}


Table S15. Calculated relative energies for 3 at the $\omega \mathrm{B} 97 \mathrm{X}-\mathrm{D} / \mathrm{BS2}(\mathrm{SMD}) / / \omega \mathrm{B} 97 \mathrm{X}-\mathrm{D} / \mathrm{BS1}$

level of theory for different spin states. The Mulliken spin population is given for cobalt, $\rho_{\mathrm{Co}}$.

${ }^{a}$ The computed energy for the spin purified triplet was obtained using Yamaguchi's approach.

\begin{tabular}{|c|c|c|c|c|c|}
\hline Multiplicity & $\left\langle\mathbf{S}^{2}\right\rangle$ & $\rho_{\text {Co }}$ & $\Delta \mathbf{E}$ & $\Delta \mathbf{Z P E}$ & $\Delta \mathbf{G}$ \\
\hline $\mathrm{S}=0$ & 1.38 & 1.06 & 0.0 & 0.0 & 0.0 \\
\hline $\mathrm{S}=1$ & 2.75 & 2.53 & $\begin{array}{c}-17.5 \\
-20.5^{a}\end{array}$ & $\begin{array}{c}-18.4 \\
-21.5^{a}\end{array}$ & $\begin{array}{c}-20.0 \\
-23.1^{a}\end{array}$ \\
\hline $\mathrm{S}=2$ & 6.01 & 2.69 & -9.3 & -9.8 & -11.2 \\
\hline
\end{tabular}

The spin contaminated triplet was corrected by using the broken spin (BS) symmetry procedure reported by Yamaguchi and co-workers: ${ }^{27-28}$

$$
{ }^{\mathrm{LS}} \mathrm{E}=\frac{{ }^{\mathrm{BS}} \mathrm{E}\left({ }^{\mathrm{HS}}\left\langle\mathrm{S}^{2}\right\rangle-{ }^{\mathrm{LS}}\left\langle\mathrm{S}^{2}\right\rangle\right)-{ }^{\mathrm{HS}} \mathrm{E}\left({ }^{\mathrm{BS}}\left\langle\mathrm{S}^{2}\right\rangle-{ }^{\mathrm{LS}}\left\langle\mathrm{S}^{2}\right\rangle\right)}{{ }_{\mathrm{HS}}\left\langle\mathrm{S}^{2}\right\rangle-{ }^{\mathrm{BS}}\left\langle\mathrm{S}^{2}\right\rangle}
$$

Yamaguchi's approach allows us to calculate the energy of the spin purified low-spin (LS) state from a high-spin state (HS) that is related to the low-spin state by spin-flip and $\left\langle\mathrm{S}^{2}\right\rangle$, which is the expected value of the total spin operator. 


\section{References}

1. Evans, D. F., The determination of the paramagnetic susceptibility of substances in solution by nuclear magnetic resonance. J. Chem. Soc. 1959, 2003.

2. Lipschutz, M. I.; Chantarojsiri, T.; Dong, Y.; Tilley, T. D., Synthesis, Characterization, and Alkyne Trimerization Catalysis of a Heteroleptic Two-Coordinate FeI Complex. J. Am. Chem. Soc. 2015, 137, 6366.

3. Bruker Bruker AXS APEX3, Bruker AXS Inc.: Madison, Wisconsin, USA, 2015.

4. Krause, L.; Herbst-Irmer, R.; Sheldrick, G. M.; Stalke, D., Comparison of silver and molybdenum microfocus X-ray sources for single-crystal structure determination. J. Appl. Crystallogr. 2015, 48, 3.

5. $\quad$ Dolomanov, O. V.; Bourhis, L. J.; Gildea, R. J.; Howard, J. A. K.; Puschmann, H., OLEX2: a complete structure solution, refinement and analysis program. J. Appl. Crystallogr. 2009, 42, 339.

6. Sheldrick, G., SHELXT - Integrated space-group and crystal-structure determination. Acta Crystallogr. A 2015, 71, 3.

7. Sheldrick, G., Crystal structure refinement with SHELXL. Acta Crystallogr. Sect. C-Cryst. Struct. Commun. 2015, 71, 3.

8. Ramadhar, T. R.; Zheng, S.-L.; Chen, Y.-S.; Clardy, J., Analysis of rapidly synthesized guest-filled porous complexes with synchrotron radiation: practical guidelines for the crystalline sponge method. Acta Crystallogr. A 2015, 71, 46.

9. $\quad$ Frisch, M. J.; Trucks, G. W.; Schlegel, H. B.; Scuseria, G. E.; Robb, M. A.; Cheeseman, J. R.; Scalmani, G.; Barone, V.; Petersson, G. A.; Nakatsuji, H.; Li, X.; Caricato, M.; Marenich, A.; Bloino, J.; Janesko, B. G.; Gomperts, R.; Mennucci, B.; Hratchian, H. P.; Ortiz, J. V.; Izmaylov, A. F.; Sonnenberg, J. L.; Williams-Young, D.; Ding, F.; Lipparini, F.; Egidi, F.; Goings, J.; Peng, B.; Petrone, A.; Henderson, T.; Ranasinghe, D.; Zakrzewski, V. G.; Gao, J.; Rega, N.; Zheng, G.; Liang, W.; Hada, M.; Ehara, M.; Toyota, K.; Fukuda, R.; Hasegawa, J.; Ishida, M.; Nakajima, T.; Honda, Y.; Kitao, O.; Nakai, H.; Vreven, T.; Throssell, K.; Montgomery Jr, J. A.; Peralta, J. E.; Ogliaro, F.; Bearpark, M.; Heyd, J. J.; Brothers, E.; Kudin, K. N.; Staroverov, V. N.; Keith, T.; Kobayashi, R.; Normand, J.; Raghavachari, K.; Rendell, A.; Burant, J. C.; Iyengar, S. S.; Tomasi, J.; Cossi, M.; Millam, J. M.; Klene, M.; Adamo, C.; Cammi, R.; Ochterski, J. W.; Martin, R. L.; Morokuma, K.; Farkas, O.; Foresman, J. B.; Fox, D. J. Gaussian 09, Rev. E.01, Gaussian, Inc., Wallingford CT, 2009.

10. Chai, J.-D.; Head-Gordon, M., Long-range corrected hybrid density functionals with damped atom-atom dispersion corrections. Phys. Chem. Chem. Phys. 2008, 10, 6615.

11. Andrae, D.; Häußermann, U.; Dolg, M.; Stoll, H.; Preuß, H., Energy-adjustedab initio pseudopotentials for the second and third row transition elements. Theor. Chim. Acta 1990, 77, 123.

12. Höllwarth, A.; Böhme, M.; Dapprich, S.; Ehlers, A. W.; Gobbi, A.; Jonas, V.; Köhler, K. F.; Stegmann, R.; Veldkamp, A.; Frenking, G., A set of d-polarization functions for pseudopotential basis sets of the main group elements Al-Bi and f-type polarization functions for $\mathrm{Zn}, \mathrm{Cd}$, Hg. Chem. Phys. Lett. 1993, 208, 237.

13. Hariharan, P. C.; Pople, J. A., The influence of polarization functions on molecular orbital hydrogenation energies. Theor. Chim. Acta 1973, 28, 213. 
14. Hehre, W. J.; Ditchfield, R.; Pople, J. A., Self-Consistent Molecular Orbital Methods. XII. Further Extensions of Gaussian-Type Basis Sets for Use in Molecular Orbital Studies of Organic Molecules. J. Chem. Phys. 1972, 56, 2257.

15. Marenich, A. V.; Cramer, C. J.; Truhlar, D. G., Universal Solvation Model Based on Solute Electron Density and on a Continuum Model of the Solvent Defined by the Bulk Dielectric Constant and Atomic Surface Tensions. J. Phys. Chem. B 2009, 113, 6378.

16. Ehlers, A. W.; Böhme, M.; Dapprich, S.; Gobbi, A.; Höllwarth, A.; Jonas, V.; Köhler, K. F.; Stegmann, R.; Veldkamp, A.; Frenking, G., A set of f-polarization functions for pseudopotential basis sets of the transition metals Sc-Cu, Y-Ag and La-Au. Chem. Phys. Lett. 1993, 208, 111.

17. Krishnan, R.; Binkley, J. S.; Seeger, R.; Pople, J. A., Self-consistent molecular orbital methods. XX. A basis set for correlated wave functions. J. Chem. Phys. 1980, 72, 650.

18. Gonzalez, C.; Schlegel, H. B., An improved algorithm for reaction path following. J. Chem. Phys. 1989, 90, 2154.

19. Gonzalez, C.; Schlegel, H. B., Reaction path following in mass-weighted internal coordinates. J. Phys. Chem. 1990, 94, 5523.

20. Grimme, S., Supramolecular Binding Thermodynamics by Dispersion-Corrected Density Functional Theory. Chem. Eur. J. 2012, 18, 9955.

21. Kozuch, S.; Shaik, S., How to Conceptualize Catalytic Cycles? The Energetic Span Model. Acc. Chem. Res. 2011, 44, 101.

22. Uhe, A.; Kozuch, S.; Shaik, S., Automatic analysis of computed catalytic cycles. J. Comput. Chem. 2011, 32, 978.

23. Bonyhady, S. J.; DeRosha, D. E.; Vela, J.; Vinyard, D. J.; Cowley, R. E.; Mercado, B. Q.; Brennessel, W. W.; Holland, P. L., Iron and Cobalt Diazoalkane Complexes Supported by $\beta$ Diketiminate Ligands: A Synthetic, Spectroscopic, and Computational Investigation. Inorg. Chem. 2018, 57, 5959.

24. Bonyhady, S. J.; Goldberg, J. M.; Wedgwood, N.; Dugan, T. R.; Eklund, A. G.; Brennessel, W. W.; Holland, P. L., Cobalt(II) Complex of a Diazoalkane Radical Anion. Inorg. Chem. 2015, $54,5148$.

25. Thom, A. J. W.; Sundstrom, E. J.; Head-Gordon, M., LOBA: a localized orbital bonding analysis to calculate oxidation states, with application to a model water oxidation catalyst. Phys. Chem. Chem. Phys. 2009, 11, 11297.

26. Shao, Y.; Gan, Z.; Epifanovsky, E.; Gilbert, A. T. B.; Wormit, M.; Kussmann, J.; Lange, A. W.; Behn, A.; Deng, J.; Feng, X.; Ghosh, D.; Goldey, M.; Horn, P. R.; Jacobson, L. D.; Kaliman, I.; Khaliullin, R. Z.; Kuś, T.; Landau, A.; Liu, J.; Proynov, E. I.; Rhee, Y. M.; Richard, R. M.; Rohrdanz, M. A.; Steele, R. P.; Sundstrom, E. J.; Woodcock, H. L.; Zimmerman, P. M.; Zuev, D.; Albrecht, B.; Alguire, E.; Austin, B.; Beran, G. J. O.; Bernard, Y. A.; Berquist, E.; Brandhorst, K.; Bravaya, K. B.; Brown, S. T.; Casanova, D.; Chang, C.-M.; Chen, Y.; Chien, S. H.; Closser, K. D.; Crittenden, D. L.; Diedenhofen, M.; DiStasio, R. A.; Do, H.; Dutoi, A. D.; Edgar, R. G.; Fatehi, S.; Fusti-Molnar, L.; Ghysels, A.; Golubeva-Zadorozhnaya, A.; Gomes, J.; Hanson-Heine, M. W. D.; Harbach, P. H. P.; Hauser, A. W.; Hohenstein, E. G.; Holden, Z. C.; Jagau, T.-C.; Ji, H.; Kaduk, B.; Khistyaev, K.; Kim, J.; Kim, J.; King, R. A.; Klunzinger, P.; Kosenkov, D.; Kowalczyk, T.; Krauter, C. M.; Lao, K. U.; Laurent, A. D.; Lawler, K. V.; Levchenko, S. V.; Lin, C. Y.; Liu, F.; Livshits, E.; Lochan, R. C.; Luenser, A.; Manohar, P.; Manzer, S. F.; Mao, S.-P.; Mardirossian, N.; Marenich, A. V.; Maurer, S. A.; Mayhall, N. J.; Neuscamman, E.; Oana, C. M.; OlivaresAmaya, R.; O’Neill, D. P.; Parkhill, J. A.; Perrine, T. M.; Peverati, R.; Prociuk, A.; Rehn, D. R.; 
Rosta, E.; Russ, N. J.; Sharada, S. M.; Sharma, S.; Small, D. W.; Sodt, A.; Stein, T.; Stück, D.; Su, Y.-C.; Thom, A. J. W.; Tsuchimochi, T.; Vanovschi, V.; Vogt, L.; Vydrov, O.; Wang, T.; Watson, M. A.; Wenzel, J.; White, A.; Williams, C. F.; Yang, J.; Yeganeh, S.; Yost, S. R.; You, Z.-Q.; Zhang, I. Y.; Zhang, X.; Zhao, Y.; Brooks, B. R.; Chan, G. K. L.; Chipman, D. M.; Cramer, C. J.; Goddard, W. A.; Gordon, M. S.; Hehre, W. J.; Klamt, A.; Schaefer, H. F.; Schmidt, M. W.; Sherrill, C. D.; Truhlar, D. G.; Warshel, A.; Xu, X.; Aspuru-Guzik, A.; Baer, R.; Bell, A. T.; Besley, N. A.; Chai, J.-D.; Dreuw, A.; Dunietz, B. D.; Furlani, T. R.; Gwaltney, S. R.; Hsu, C.-P.; Jung, Y.; Kong, J.; Lambrecht, D. S.; Liang, W.; Ochsenfeld, C.; Rassolov, V. A.; Slipchenko, L. V.; Subotnik, J. E.; Van Voorhis, T.; Herbert, J. M.; Krylov, A. I.; Gill, P. M. W.; Head-Gordon, M., Advances in molecular quantum chemistry contained in the Q-Chem 4 program package. Mol. Phys. 2015, 113, 184.

27. Soda, T.; Kitagawa, Y.; Onishi, T.; Takano, Y.; Shigeta, Y.; Nagao, H.; Yoshioka, Y.; Yamaguchi, K., Ab initio computations of effective exchange integrals for $\mathrm{H}-\mathrm{H}, \mathrm{H}-\mathrm{He}-\mathrm{H}$ and $\mathrm{Mn}_{2} \mathrm{O}_{2}$ complex: comparison of broken-symmetry approaches. Chem. Phys. Lett. 2000, 319, 223.

28. Yamaguchi, K.; Jensen, F.; Dorigo, A.; Houk, K. N., A spin correction procedure for unrestricted Hartree-Fock and Møller-Plesset wavefunctions for singlet diradicals and polyradicals. Chem. Phys. Lett. 1988, 149, 537. 